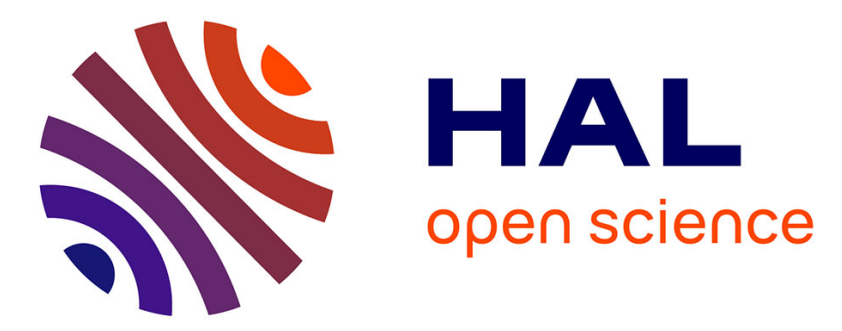

\title{
La survenue du cancer : effets de court et moyen termes sur l'emploi, le chômage et les arrêts maladie
} Thomas Barnay, Mohamed Ben Halima, Emmanuel Duguet, Joseph Lanfranchi, Christine Le Clainche

\section{- To cite this version:}

Thomas Barnay, Mohamed Ben Halima, Emmanuel Duguet, Joseph Lanfranchi, Christine Le Clainche. La survenue du cancer: effets de court et moyen termes sur l'emploi, le chômage et les arrêts maladie. Economie et Statistique / Economics and Statistics, 2015. hal-01297568

\section{HAL Id: hal-01297568 https://hal.science/hal-01297568}

Submitted on 4 Apr 2016

HAL is a multi-disciplinary open access archive for the deposit and dissemination of scientific research documents, whether they are published or not. The documents may come from teaching and research institutions in France or abroad, or from public or private research centers.
L'archive ouverte pluridisciplinaire HAL, est destinée au dépôt et à la diffusion de documents scientifiques de niveau recherche, publiés ou non, émanant des établissements d'enseignement et de recherche français ou étrangers, des laboratoires publics ou privés. 


\title{
La survenue du cancer : effets de court et moyen termes sur l'emploi, le chômage et les arrêts maladie
}

\author{
Thomas Barnay *, Mohamed Ali Ben Halima **, Emmanuel Duguet ***, \\ Joseph Lanfranchi **** et Christine Le Clainche *****
}

La réduction des inégalités face à la maladie est un des attendus majeurs du troisième Plan cancer 2014-2019, qui préconise de " diminuer l'impact du cancer sur la vie personnelle » afin d'éviter la « double peine » (maladie et exclusion du marché du travail).

Dans ce contexte, nous évaluons l'impact de un à cinq ans d'un primo-enregistrement en Affection de longue durée (ALD) caractérisant le cancer sur la situation professionnelle et la durée passée en emploi, maladie et chômage de salariés du secteur privé. Nous utilisons la base de données administratives Hygie, recensant la carrière professionnelle et les épisodes de maladie d'un échantillon de salariés affiliés au Régime général de la Sécurité sociale. L'évaluation de l'impact de la survenue du cancer s'appuie sur une méthode de double différence avec appariement exact pour comparer les salariés malades aux salariés sans aucune ALD.

La première année après le diagnostic correspond au temps des traitements caractérisé par une augmentation du nombre de trimestres d'arrêts de travail pour maladie de 1,7 pour les femmes et de 1,2 pour les hommes. L'âge joue également un rôle sur les absences liées à la maladie. Par ailleurs, l'employabilité des travailleurs atteints du cancer diminue avec le temps. La proportion de femmes et d'hommes employés au moins un trimestre, baisse respectivement de 8 et 7 points de pourcentage dans l'année suivant la survenue du cancer et jusqu'à 13 points de pourcentage cinq ans plus tard. Cette distance à l'emploi se renforce lorsque les salariés malades sont plus âgés. L'effet de la maladie à cinq ans est respectivement de 15 et 19 points de pourcentage pour les hommes de plus de 51 ans et pour les femmes de plus de 48 ans.

Ces différences de genre et d'âge peuvent traduire des différences de localisation et de sévérité des cancers, d'une part, de séquelles des cancers et de difficultés de réinsertion sur le marché du travail plus importantes avec l'avancée en âge, d'autre part.

Rappel :

Les jugements et opinions exprimés par les auteurs n'engagent qu'eux mêmes, et non les institutions auxquelles ils appartiennent, ni a fortiori l'Insee.
Codes JEL : I1, J21, J22.

Mots clés : cancers, situation professionnelle, méthode de différences des différences avec appariement exact.

\footnotetext{
* Université de Rouen, Cream ; Érudite (EA 437), UPEC, UPEM, TEPP(FR CNRS 3435).

** Irdes, chercheur associé au Centre d'étude de l'emploi (CEE).

*** Université Paris Est, Érudite (EA 437), UPEC, UPEM, TEPP(FR CNRS 3126) et CEE.

**** Université Paris 2, LEMMA, CEE.

${ }^{* * * * *}$ ENS Cachan, CES Cachan, CEE. Auteur correspondant : christine.leclainche@ens-cachan.fr.
}

La base de données Hygie, utilisée dans cet article, a été construite par l'Institut de recherche et documentation en économie de la santé (Irdes) en réponse à un appel d'offre de la Direction de la recherche, des études, de l'évaluation et des statistiques (Drees), à l'aide des données fournies par la Caisse nationale d'assurance vieillesse (Cnav) et de la Caisse nationale d'assurance maladie des travailleurs salariés (Cnamts).

Nos remerciements vont à Martine Audibert (Centre d'étude et de recherche sur le développement international, Cerdi), Université de Clermont-Ferrand), aux participants à la $10^{\circ}$ conférence annuelle Travail, emploi et politiques publiques (Tepp) et aux $35^{\mathrm{e}} \mathrm{Journées} \mathrm{des}$ économistes de la santé français pour leurs remarques constructives. Nous remercions également Camille Regaert et Catherine Sermet de I'Irdes impliquées plus particulièrement dans d'autres parties de cette recherche. Ce travail doit également beaucoup aux commentaires avisés d'Ève Caroli, Chantal Cases, Laurence Chérié-Challine, Fatema Colonna, Jean-Michel Domergue, Paul Dourgnon, Agnès Rogel et Anne Thuret. Enfin, nous remercions l'ARC et l'Institut national du cancer (Inca) pour le soutien financier apporté à cette recherche. 
D epuis le premier Plan cancer (2003-2007), la lutte contre le cancer est un enjeu de santé publique majeur en France. La croissance de l'incidence du cancer est de près de $109 \%$ entre 1980 et 2012, année durant laquelle 355354 nouveaux cas ont été détectés, dont 200350 chez les hommes et 155004 chez les femmes. L'âge médian au diagnostic était en 2012 de 68 ans pour les hommes et 67 ans pour les femmes (Inca, 2015).

L'allongement de la durée de cotisation requise pour l'obtention d'une retraite à taux plein, engagé depuis la réforme de $1993^{1}$, combiné à un dépistage plus précoce et souvent organisé des cancers, conduit de plus en plus de malades du cancer à être sur le marché du travail au moment de la survenue de la maladie, avec un horizon de carrière potentiel assez long.

Selon les modèles de capital santé, la survenue du cancer, comme tout événement de santé grave, affecte la trajectoire professionnelle via des effets potentiels sur le stock (choc exogène instantané) et sur le taux de dépréciation du capital de santé mais aussi, plus globalement, sur les investissements futurs en capital humain (actions de prévention primaire ou secondaire en santé). La maladie peut notamment conduire les individus à une réallocation du temps utilisé entre travail et temps non travaillé. Afin de recevoir les traitements longs, le patient doit tout d'abord libérer du temps de travail et procéder à une réallocation temporelle en faveur du temps non marchand. Selon la flexibilité offerte par le contrat de travail (Contrat à durée indéterminée (CDI) / Contrat à durée déterminée (CDD), temps plein / partiel...) et les possibilités d'aménagement du poste, l'offre de travail, le cas échéant, peut être adaptée, voire réduite. Par ailleurs, la survenue du cancer et les traitements de longue durée risquent d'affecter durablement la productivité individuelle de salariés souvent âgés de plus de 45 ans. Le risque d'exclusion temporaire, voire définitif, du marché du travail s'accroît sensiblement dès 50 ans. Même s'il croît, le taux de retour à l'emploi des chômeurs âgés de 50 à 54 ans reste seulement de $27 \%$ sur la période 2009-2011 (Govillot et Rey, 2013). Le risque de sortie définitive du marché du travail pour les personnes souffrant de cancer n'est donc pas négligeable lorsqu'elles sont jugées inaptes au poste de travail occupé avant la maladie.

L'objectif de cet article est d'étudier l'effet de la survenue d'un cancer, identifiée par le primo-enregistrement administratif en Affection de longue durée (ALD), sur la trajectoire professionnelle. Nous considérons la survenue du cancer comme un choc exogène de santé. Cette hypothèse mérite une clarification. Bien que le cancer soit en partie lié à des comportements à risque (notamment tabac, alcool) ou à des expositions à des cancérogènes, la base de données mobilisée concerne une population plutôt jeune pour laquelle les cas de cancers détectés sont moins susceptibles d'être mis en relation avec des comportements à risque. En effet, le développement de cancers à la suite d'exposition à des facteurs de risque est différé dans le temps, le cancer demeurant une maladie du vieillissement ${ }^{2}$. En outre des travaux récents qui, cependant n'incluent pas les cancers du sein et de la prostate, montrent que les cancers seraient principalement dus à la malchance plutôt qu'à des facteurs génétiques ou à des facteurs de comportements à risque ou d'environnement (de l'ordre de deux tiers pour la malchance et d'un tiers pour les autres facteurs, voir Tomasetti, Vogelstein, 2015).

De ce fait, nous considérons que le cancer peut être interprété comme un choc exogène qui vient affecter la possibilité de se maintenir sur le marché du travail ou la trajectoire d'emploi. Ceci traduit en partie la modification des arbitrages entre temps travaillé et temps non travaillé comme conséquence du cancer y compris à long terme du fait de la présence de séquelles, de motivations de préservation de sa santé etc.

Contrairement aux autres travaux français dont l'objectif est de mesurer l'effet à deux ans de la survenue du cancer (Duguet et Le Clainche, 2012a; Eichenbaum-Voline et al., 2008 ; Joutard et al., 2012), nous menons une analyse prospective plus longue permettant de mesurer des phénomènes de persistance du choc de santé. Nous apprécions ainsi les effets de court (à un an) et moyen termes (de deux à cinq ans $)^{3}$ de

\footnotetext{
1. Par ailleurs, depuis 2010 , l'âge légal de la retraite est porté progressivement de 60 ans à 62 ans.

2. Dans les pays occidentaux, les trois quarts des cancers apparaissent après 55 ans. Plus de la moitié est détectée après 65 ans (voir Inca 2015).

3. Nous considérons par convention que le court terme correspond à une durée d'un an après le diagnostic et le moyen terme à une durée variant de deux à cinq ans. Dans beaucoup de maladies cancéreuses, une durée d'un an signale le temps des traitements et marque l'échéance à partir de laquelle la personne traitée peut reprendre des activités normales incluant des activités professionnelles. Une échéance de deux ans est quant à elle associée aux chances de survie à court terme : une fois passé ce cap, sans récidive, le patient est considéré en rémission. Au-delà de cinq ans après le diagnostic et sans reprise de la maladie, les risques de récidive sont supposés se réduire de manière importante. Bien évidemment, il existe une variabilité importante concernant l'échéance temporelle à partir de laquelle la rémission complète peut être constatée, selon les organes cibles du cancer et son agressivité.
} 
la survenue du cancer sur le marché du travail. Afin de décrire précisément la situation sur le marché du travail en termes qualitatifs et quantitatifs, nous construisons des indicatrices de situation et de durée relatives à l'emploi, au chômage et aux arrêts maladie.

Ce double objectif (temporalité longue et relevé précis des situations face au marché du travail post cancer) est conditionné par la mobilisation d'une base de données administratives riche. Pour ce faire, nous utilisons la base Hygie structurée en panel à partir de 2005 et qui permet le recueil, pour les individus du secteur privé échantillonnés, d'une information rétrospective exhaustive des carrières professionnelles tout en renseignant certaines informations médicales et notamment en termes d'inscription dans le régime de sécurité sociale dit des Affections de longue durée (ALD) pour les salariés du secteur privé. Pour isoler l'effet propre du cancer, nous avons recours à une méthode de double différence avec appariement exact pour comparer les salariés malades à leurs « jumeaux », définis comme des salariés sans aucune ALD, dont les caractéristiques socio-économiques et le statut professionnel antérieur au choc de santé sont identiques au salarié malade.

L'article est structuré de la manière suivante : la première section présente des éléments de revue de littérature et souligne l'originalité de l'approche retenue, la deuxième section décrit la base de données et la méthode économétrique utilisée puis les sections suivantes présentent et discutent les résultats.

\section{Une littérature internationale foisonnante mais limitée en France}

Du fait de l'augmentation de la survie et des possibilités de retour au travail, le cancer peut s'apparenter à une maladie chronique au regard de ses conséquences sur l'emploi et les revenus.

L'effet de la maladie chronique sur le marché du travail peut relever de mécanismes liés à l'offre ou à la demande de travail. Les analyses relatives à la discrimination produites par Becker (1957, 1971), Arrow (1973) et Phelps (1972), par exemple, peuvent également être utiles pour comprendre comment l'insertion dans l'emploi va être affectée par la maladie.

Dans la mesure où une santé précaire aura un effet négatif sur la productivité, les individus en mauvaise santé auront une plus faible probabilité d'être employés au taux de salaire du marché. Par ailleurs, la baisse des rémunérations due à la plus faible productivité peut modifier, en l'absence de mécanismes financiers compensatoires, les coûts d'opportunité associés au loisir, à tout le moins associés au temps non travaillé, et partant, modifier la participation au marché du travail. Il est également possible qu'en dehors même de la médiation de la productivité, la relation entre santé et offre de travail soit modifiée du fait que l'utilité associée au revenu et au temps passé en dehors du marché du travail est elle-même affectée indépendamment de la productivité de l'individu. Le temps non professionnel peut, en effet, être utilisé pour se soigner.

Enfin, différents phénomènes de discrimination peuvent être à l'œuvre. Si les employeurs croient ou constatent qu'en général les individus malades sont moins productifs, ils peuvent adopter un comportement discriminatoire à l'embauche ou à la promotion de ces personnes (il s'agit alors de discrimination statistique, voir Arrow, 1973, Phelps, 1972). Les personnes malades, ou ayant été malades, peuvent alors intégrer de telles croyances et modifier leur offre de travail, en anticipant par exemple leur sortie du marché du travail (Coate et Loury, 1993).

Une discrimination pure pourra également se produire si les employeurs ont une préférence pour ne pas embaucher ou intégrer dans l'entreprise des individus ayant été malades ou confrontés à une maladie. Il existe toutefois certains outils de lutte contre la discrimination sur le marché du travail français telles les modalités de déclaration des inaptitudes partielles ou totales par la médecine du travail régies par l'article R 241-5 du code du travail. En dépit de ces mesures, les personnes ayant des problèmes de santé ou un handicap déclarent plus fréquemment subir des comportements de stigmatisation sur leur lieu de travail (Bouvier et Jugnot, 2013).

Plusieurs spécificités du cancer expliquent son caractère plus invalidant sur le marché du travail que la plupart des maladies chroniques. En raison de traitements souvent lourds, le cancer a des séquelles potentiellement majeures qui peuvent renforcer l'inaptitude à l'occupation de certains postes de travail par les individus. Des comorbidités associées directement à certains traitements reçus peuvent également se produire. Enfin le cancer peut être caractérisé par des épisodes de récurrence avec de nouveaux traitements parfois plus agressifs que ceux 
précédemment reçus, ce qui peut produire ou amplifier des inaptitudes.

Ces similitudes et différences avec le cas des maladies chroniques doivent être relevées afin de renseigner les analyses de l'effet à court et moyen termes du cancer sur la situation professionnelle.

La littérature empirique sur les liens entre cancer et emploi montre que l'effet de la survenue du cancer est négatif sur la participation au marché du travail, la probabilité d'emploi et les heures travaillées. Dans une méta-analyse fondée sur vingt-six articles (sur données américaines et européennes), de Boer et al. (2009) estiment par exemple que le risque relatif de chômage des survivants du cancer est égal à 1,37 en référence à une population en bonne santé, toutes choses égales par ailleurs. Les principales observations statistiques disponibles pour la France viennent de l'exploitation de « l'enquête nationale sur les conditions de vie des personnes atteintes d'une maladie longue ou chronique » réalisée par la Drees et l'Inserm sur un ensemble de survivants $\mathrm{du}$ cancer deux ans après leur enregistrement en ALD $^{4}$. Selon cette source, seuls $66 \%$ des survivants du cancer, en emploi au moment du diagnostic, le sont encore deux ans plus tard (Paraponaris et al., 2010).

Mehnert (2011), dans une revue de littérature référençant soixante quatre articles internationaux, indique que le retour à l'emploi des survivants du cancer est, en moyenne selon les études, de $40 \%$ six mois après le diagnostic, $62 \%$ après douze mois, $73 \%$ après dix-huit mois et $89 \%$ après vingt-quatre mois. Inversement, la survenue d'un cancer favorise une sortie définitive du marché du travail. À âge et sexe donnés, le risque relatif associé au départ anticipé en retraite est de 2,2 pour les survivants du cancer du système nerveux, de 2 pour la leucémie, de 1,9 pour la langue, de 1,2 pour le sein et de 1,06 pour la prostate (Taskila-Abrandt et al., 2005).

L'effet négatif du cancer sur la trajectoire professionnelle transite majoritairement par le biais des limitations fonctionnelles (Bradley et al., 2002) qui peuvent être spécifiques telles que les douleurs au bras pour le cancer du sein (Quinlan et al., 2009; Blinder et al., 2012) mais aussi des épisodes dépressifs (Damkjaer et al., 2011), des troubles de concentration et de mémoire (Oberst K. et al., 2010) et plus globalement des risques psychosociaux plus marqués (Cooper et al., 2012). Ces dynamiques sont plus ou moins amplifiées selon la nature des dotations initiales en capital humain (niveau de formation), la pénibilité physique de l'emploi occupé avant la maladie, l'origine ethnique, mais aussi le type de cancer (site, sévérité de la maladie) et enfin la nature des traitements (Mujahid et al., 2011 ; Drolet et al., 2005 ; Lindbohm et al., 2011 ; Johnsson et al., 2011 ; Blinder et al., 2012).

Les caractéristiques de la carrière antérieure à la survenue du cancer sont rarement introduites pour mesurer l'effet d'un choc exogène de santé sur le parcours professionnel. Pourtant, les épisodes d'interruptions d'emploi et de chômage peuvent entrainer des effets stigmatisants sur la carrière (Heckman et Borjas, 1980 ; Gregg et Tominey, 2005) et la santé des individus et, pour certaines professions, prédire la survenue de cancers professionnels. Enfin, l'un des facteurs limitant les possibilités de maintien et de retour au travail après le diagnostic de cancer est la discrimination ressentie sur le lieu de travail. En France, Paraponaris et al. (2010) rapportent que les survivants du cancer deux ans après le diagnostic souffrent de tels comportements discriminatoires de la part de collègues ou de la hiérarchie. Toutes choses égales par ailleurs, ces comportements augmentent de $15 \%$ la probabilité qu'ils perdent leur emploi.

L'environnement de travail influence également la qualité de l'emploi après la survenue de la maladie. Dans leur revue de littérature, Feuerstein et al. (2010) insistent sur l'importance des aménagements du milieu de travail en termes de flexibilité des horaires, de soutien des collègues, de climat social et de stress. Duguet et Le Clainche (2012 a) montrent que la probabilité de retour au travail de salariés français deux ans après le diagnostic du cancer s'accroît lorsque les conditions de travail ont été adaptées.

La littérature sur les conséquences du cancer a également analysé les effets de genre avant tout liés à la spécificité des cancers.

Dans la littérature consacrée à l'effet du cancer sur les absences au travail, un certain nombre de travaux ont ainsi permis de faire des constats variables selon le contexte institutionnel et économique inhérent à chaque pays et selon les échéances temporelles ciblées pour l'analyse des résultats sur le marché du travail, à partir du diagnostic. Dans le contexte américain, par

4. Certaines études ont toutefois été réalisées sur des échantillons locaux de taille plus réduite, pour des types de cancers spécifiques (voir par exemple l'étude de Fantoni et al., 2010, sur le retour au travail après un cancer du sein dans le nord de la France). 
exemple, Bradley et al. (2006) montrent que la survenue d'un cancer du sein est associée à 45 jours d'absence au travail contre 27 jours à la suite d'un cancer de la prostate, ceteris paribus. Lorsque l'ensemble des cancers est pris en compte, les résultats sur l'offre de travail selon le genre ne sont pas forcément convergents. Ainsi, une étude en population générale réalisée en Corée sur les 21 cancers les plus fréquents montre que si les patientes ont plus de risque de perdre leur emploi que leur homologues masculins, elles ont également une probabilité plus élevée d'être réemployées six ans après le diagnostic (Park et al, 2008). Les différences de résultats selon le genre semblent également liées à l'apparition de nouveaux cancers et à l'horizon temporel d'analyse.

Moran et al. (2011) s'intéressent à un panel d'Américains âgés de 28 à 54 ans et à tous types de cancers en mobilisant entre autres méthodes, celle du score de propension et des doubles différences. À moyen terme (horizon de deux à six ans), la survenue d'un nouveau cancer ou d'une récidive conduit à une diminution des taux d'emploi féminins de 19 à 21 points de pourcentage contre 28 à 30 points chez les hommes ${ }^{5}$. En revanche, lorsque l'on considère tous les malades, avec ou sans nouveau cancer, les écarts entre les hommes et les femmes disparaissent; les survivants du cancer ont des taux d'emploi de 7 à 8 points de pourcentage inférieurs à ceux des salariés en bonne santé. En France, le taux de retour à l'emploi deux ans plus tard n'est pas significativement plus élevé pour les hommes que les femmes (Malavolti et al., 2008 ; Marino et al., 2013) ${ }^{6}$. Toutefois, des différences apparaissent dans la vitesse de retour à l'emploi puisque $38 \%$ des femmes et $53 \%$ des hommes sont de retour sur le marché du travail après six mois. Ce n'est qu'au bout de dix-huit mois que les taux de retour à l'emploi convergent. Si les types de traitements reçus expliquent une partie de ces écarts, le retour plus rapide au travail des hommes renverrait également, selon les auteurs, à la plus forte pression sociale sur le retour au travail (voir Crompton, 1999, pour un modèle du male breadwinner). Ainsi, les hommes mariés retournent en emploi beaucoup plus rapidement que les femmes mariées.

Par ailleurs, les études sur les effets de la survenue du cancer sur l'emploi se sont majoritairement intéressées aux personnes de plus de 50 ans. Les cancers les plus courants sont en effet des cancers survenant souvent au-delà de cet âge (sein, prostate, côlon). Néanmoins, l'augmentation des diagnostics de cancers précoces dans le cycle de vie nécessite d'étudier le rôle de la survenue de la maladie sur la situation professionnelle pour des individus jeunes ${ }^{7}$. Eichenbaum-Voline et al. (2008) montrent que deux ans après l'enregistrement en ALD cancer, les sorties de l'emploi sont plus fréquentes pour les personnes de plus de 50 ans. Une partie de cette population se dirige naturellement vers la retraite, mais leur sortie vers l'inactivité est également plus importante que celle des populations plus jeunes. Enfin, les individus de plus de 50 ans se trouveraient plus fréquemment contraints que les plus jeunes à des situations de chômage.

Si la littérature internationale apparaît foisonnante, peu d'études sont toutefois consacrées aux effets au-delà de deux ans de la survenue d'un cancer, sur la situation vis-à-vis du marché du travail. En outre, les travaux économiques en France sont encore rares. De nombreuses questions restent donc à étudier.

Parmi ces questions, les effets de genre et d'âge établis dans la littérature doivent être confirmés par le recours à des méthodes économétriques plus robustes. En effet, parmi les études françaises citées, certaines ne peuvent bénéficier de groupes de contrôle pour estimer les conséquences du diagnostic de cancer quant au maintien en emploi ou au retour à l'emploi, comparativement à la situation des individus non concernés par la maladie ${ }^{8}$. Il reste aussi à déterminer si l'étude d'horizons temporels plus longs (deux ans pour les études les plus récentes en France), confirment (ou non) l'effet différencié par genre observé selon l'horizon pour les cancers les plus couramment étudiés et qui sont aussi associés à des survies plus longues.

Notre analyse se propose par conséquent d'approfondir la connaissance de l'effet de la survenue d'un cancer sur la situation vis-à-vis du

5. En outre, les taux d'emploi à plein temps diminuent de 16 à 23 points de pourcentage et le nombre d'heures travaillées de $26 \%$ chez les femmes survivantes contre respectivement de 28 à 30 points et de $38 \%$ chez les hommes survivants.

6. Les travaux portent sur la base de données Drees-Inserm, issue de l'enquête "La vie deux ans après un cancer " réalisée en 2004 et traitent de tous les cancers. Le cancer du sein est le cancer le plus prévalent chez les femmes dans cette base comme dans les données d'incidence et de prévalence (du fait de la survie associée à ce cancer).

7. Rares sont les études ciblant plus spécifiquement les liens entre cancer et statut d'occupation pour une population plus jeune (cf. Moran et al., op. cit., Taskila-Abrandt et al., 2005).

8. Seules les études de Eichenbaum-Voline et al. (2008) et Joutard et al. (2012), apparient les salariés malades de l'enquête sur les conditions de vie des personnes atteintes d'une maladie longue avec les salariés de l'enquête Emploi. Selon les échantillons et les méthodes utilisés, ces auteurs chiffrent l'écart de probabilité de maintien en emploi à deux ans entre $12,6 \%$ et $14,2 \%$. 
marché du travail en calculant de manière précise et sur cinq années la situation sur le marché $\mathrm{du}$ travail et le nombre de trimestres passés en arrêt maladie, en emploi ou au chômage, selon le genre et l'âge lors du premier enregistrement en ALD cancer.

\section{Source et méthode}

C oncernant les bases de données utilisées dans la littérature, la plupart des travaux s'intéressant au rôle du cancer sur les trajectoires professionnelles s'appuient sur des données de registre national du cancer, de maladies chroniques (comme au Danemark ou en Norvège) ou issues de centres de traitement ou d'hôpitaux (comme à Baltimore en Pennsylvanie ; cf. Moran et al., op. cit.). Les trois quarts des études que nous avons répertoriées sur le sujet depuis 2000 mobilisent ainsi des données administratives sur la maladie mais également sur les caractéristiques socio-économiques et professionnelles des individus. Le recours à ce type de données évite les biais de déclaration et de mémoire caractéristiques des données déclaratives. Dans la plupart de ces travaux cependant, les échantillons d'études sont de petite taille, les données cliniques collectées étant ensuite appariées à des données individuelles issues de bases administratives.
L'originalité de notre étude consiste notamment à recourir à une base administrative comportant des données rétrospectives de carrière.

\section{Présentation de la base Hygie}

Nous utilisons la base Hygie 9 qui est constituée de données administratives issues de la Caisse nationale d'assurance vieillesse (Cnav) et de la Caisse nationale d'assurance maladie des travailleurs salariés (Cnamts) (cf. encadré 1). Elle fournit des informations individuelles sur les bénéficiaires (le sexe, l'âge, le statut d'occupation...), leur biographie professionnelle complète dans le Régime général (trimestres validés en emploi, chômage ou retraite et historique annuel des salaires), leurs consommations médicales courantes (en volume, en dépenses annuelles réelles et remboursées), leurs arrêts de travail (maladie et accidents du travail (AT)/ maladies professionnelles (MP), indemnités journalières) et une information détaillée sur les ALD (numéro d'ALD, ancienneté de l'enregistrement ALD, exonérations pour maladie professionnelle, nature médicale précise...).

9. Pour plus d'informations relatives à la base Hygie, une description détaillée est disponible à cette adresse: http://www. Irdes.fr/EspaceRecherche/Partenariats/Hygie/index.htm.

Encadré 1

\section{HYGIE : UNE SOURCE DE DONNEES MÉDICO-ADMINISTRATIVES SUR LES PARCOURS PROFESSIONNELS}

Les données Hygie sont issues des bases administratives de la Caisse nationale d'assurance vieillesse (Cnav) et de la Caisse nationale d'assurance maladie des travailleurs salariés (Cnamts) et se rapportent exclusivement au secteur privé. Plus spécifiquement, les bases de données de la Cnav proviennent du Système national de gestion des carrières (SNGC) qui regroupe les salariés et du Système national statistiques prestataires (SNSP) constitué des retraités. Ces données sont ensuite appariées aux prestations d'assurance maladie (Cnamts) tirées du Système national d'information inter-régimes de l'Assurance maladie (Sniir-am). Les données de la Cnav constituent donc le point d'entrée de la constitution de l'échantillon. II s'agit d'un échantillon aléatoire de bénéficiaires âgés de 22 à 70 ans en 2005 et ayant cotisé au moins une fois au Régime général de retraite au cours de leur vie. II est couplé aux données du Sniir-am qui portent sur les assurés (ouvrants droit ou ayants droit) du Régime général d'assurance maladie et pour lesquels l'Assurance maladie a enregistré au moins une prestation lors des années 2003, 2004 ou 2005.
Ces prestations médicales renseignent, par exemple, les visites auprès des généralistes ou spécialistes, les consommations médicales, les indemnités journalières liées à un arrêt maladie, un accident de travail ou Affection de longue durée (ALD), etc. Les données de carrière portent sur 552048 actifs auprès du Régime général et retraités en 2005. Les données appariées Cnav/Cnamts représentent $96,7 \%$ de la population initiale (Seuls 18128 individus appartenant au fichier de la Cnav ne sont pas appariés avec les données de la Cnamts).

La distribution par genre des populations appariée et non appariée entre les fichiers administratifs de la Cnav et les données du Sniir-am de la Cnamts est assez similaire. Les hommes représentent $42,9 \%$ de la population non appariée contre $52,1 \%$ pour l'échantillon des appariés. Cependant, l'analyse selon l'âge montre que la population non appariée Cnav/Cnamts est une population plus âgée que celle des appariés. En 2008, $56,6 \%$ des individus de la population non appariée appartiennent à la classe d'âge des $64-73$ ans contre uniquement $14,6 \%$ de la population appariée. 
Nous identifions les individus malades d'un cancer par l'enregistrement en ALD cancer Tumeur maligne, affection maligne du tissu lymphatique ou hématopoïétique (encadré 2).

Le système actuel de repérage des cancers repose sur différentes sources avec, notamment, les registres des cancers, qui sont partiels puisque ciblés mais exhaustifs sur quelques départements (ils couvrent ainsi $20 \%$ de la population). Les données nationales de mortalité du Centre d'épidémiologie sur les causes médicales de décès (CepiDc-Inserm) permettent, pour leur part, de recenser la mortalité par cancer. Les bases ALD garantissent une couverture nationale. Néanmoins, elles ne permettent pas un recensement exhaustif des cas de cancers. Par exemple, certains cancers ne sont pas systématiquement enregistrés en ALD cancer, c'est le cas notamment des mélanomes avec des risques très limités de récidives. De même, certains cancers in situ sont enregistrés en ALD cancer tandis que d'autres non (Danzon et al., 2012). Par ailleurs, la cohérence de l'incidence des cancers par l'usage des données de base ALD cancer avec d'autres bases utilisées à des fins épidémiologiques (comme les registres du cancer) est très différente selon les types de cancers (Grosclaude et al., 2012). L'interprétation de nos résultats, notamment sur le genre, doit être prudente du fait de l'existence d'un écart de sensibilité sur l'incidence des cancers tels que repérés en ALD cancer.

La base utilisée permet une couverture importante de la population d'individus salariés du secteur privé, ciblée ici sur les cotisants du Régime général, du Régime général vieillesse et de l'Assurance maladie. L'incidence des cancers nous intéresse plus particulièrement car nous souhaitons évaluer l'effet de la survenue d'un cancer sur l'emploi. La survenue d'un cancer est ainsi une notion proche de l'incidence mesurée à partir du cumul des survenues au cours d'une période donnée. Sans être en mesure de rendre compte du diagnostic de cancer ni du (ou des) traitement(s) mis en place pour y faire face, l'ancienneté dans l'ALD cancer et l'âge au diagnostic sont disponibles ainsi que les co-morbidités en ALD.

\section{Stratégie économétrique}

La méthode de différences des différences avec appariement exact vise à estimer l'effet propre de la survenue du cancer sur les situations

\section{Encadré 1 (suite)}

L'appariement permet de construire le panel de la base Hygie composé de 533920 bénéficiaires, dont $47,6 \%$ de femmes.

Pour notre étude, nous utilisons les informations de la carrière issues des données administratives de la Cnav. Ces données retracent l'ensemble de la carrière professionnelle des individus échantillonnés en 2005 depuis leur entrée sur le marché du travail. Ainsi nous disposons de leur biographie professionnelle complète associée à l'inscription au Régime général (trimestres validés en emploi, chômage, maladie ou retraite et historique annuel des salaires).

Afin d'identifier les individus malades d'un cancer, nous utilisons les informations disponibles dans le fichier relatif aux données médico-administratives de la Cnamts de 2005 à 2008. Ces données fournissent des informations sur les ALD (numéro d'ALD, date de début d'ALD qui peut être antérieur à 2005 , ancienneté de l'ALD, détail des exonérations pour maladie professionnelle, nature médicale précise...). Au total, 79933 individus ont à un moment donné souffert d'une ALD dont 13981 d'une ALD cancer (soit 17,5\% de l'échantillon des ALD contre $19 \%$ d'après les chiffres de la Cnamts). Les femmes représentent $55 \%$ de la population souffrant ou ayant souffert d'une ALD cancer.
Les individus atteints d'un cancer et bénéficiant d'une déclaration en ALD cancer peuvent avoir une date de déclaration en ALD antérieure à 2005. La date de déclaration d'ALD est une date de diagnostic (traitement statistique). Concernant l'ancienneté de l'ALD cancer, $15 \%$ des bénéficiaires d'une ALD cancer sont déclarés avant 1995, 58,8 \% entre 1995 et 2004 et $25,8 \%$ des bénéficiaires d'une ALD cancer ont une date de déclaration située entre 2005 et 2008. Cependant, l'ancienneté de l'ALD n'est pas une variable de l'analyse. En effet, les règles d'enregistrement des pathologies en ALD ont connu d'importantes modifications au fil du temps qui ne nous permettent pas de connaître précisément la durée des ALD, en particulier pour les personnes les plus âgées de notre base et ayant eu un enregistrement en ALD à un âge jeune. Depuis quelques années et notamment la loi du 13 août 2004 , les règles sont devenues plus rigoureuses et l'enregistrement en ALD est revu périodiquement, la plupart du temps tous les cinq ans.

L'appariement de ces données médicales et des carrières professionnelles fournit le moyen d'étudier l'effet du cancer sur la carrière professionnelle à court et moyen termes par rapport à la date d'enregistrement en ALD. 
professionnelles postérieures ${ }^{10}$. Elle permet d'éliminer l'effet des différentes sources d'hétérogénéité individuelle, qu'elle soit observable ou non mais invariante dans le temps d'une part, et temporelle d'autre part.

Elle consiste, dans un premier temps, à apparier les individus sur les variables observables afin d'éliminer leurs effets sur la situation professionnelle et le nombre de trimestres validés (emploi salarié, arrêt maladie, chômage). La population étudiée souffre d'un cancer (dont la date de survenue est définie par le premier enregistrement en ALD cancer). Elle est appariée à une population témoin ne souffrant d'aucune ALD et dont les caractéristiques observables, indépendamment de la survenue du cancer, sont identiques. La période sur laquelle sont observés les individus fait également partie des critères d'appariement : neutraliser les effets de la conjoncture économique, par exemple, nécessite que chaque individu cancéreux soit comparé à des individus témoins suivis sur exactement la même période de temps.
Habituellement, le nombre total d'observations de la base de données utilisée limite les possibilités de trouver pour chaque individu son équivalent du point de vue des variables observables et sur la même fenêtre d'observation. La taille de la population couverte par la base de données Hygie autorise la mise en place d'un appariement exact des individus. En d'autres termes, la population témoin est choisie pour avoir exactement les mêmes caractéristiques que la population traitée. Par ailleurs, à un individu traité correspondent plusieurs jumeaux. Nous prenons alors le résultat moyen de tous les jumeaux de chaque individu traité comme point de comparaison.

Dans un second temps, on effectue la différence entre la situation professionnelle d'un individu de la population des malades avant et après le cancer. Sont ainsi éliminés les effets de l'hétérogénéité individuelle inobservable, qui peuvent être liés par exemple à des

10. La présentation intuitive de cette méthode proposée ici est inspirée par l'article de Duguet et Le Clainche (2012b). Les détails techniques sont présentés dans l'encadré 3.

\section{Encadré 2}

\section{AFFECTIONS DE LONGUE DUREE (ALD) : RECONNAISSANCE ET PRISE EN CHARGE}

Les Affections de longue durée (ALD) sont des maladies qui nécessitent des soins longs et des traitements coûteux. II est important de différencier les ALD des maladies chroniques. Si une ALD est quasiment tout le temps une maladie chronique, un certain nombre de maladies chroniques ne sont pas des ALD (c'est le cas, par exemple, de l'arthrose et du glaucome). La reconnaissance de l'ALD (Articles L322-3 et D322-1 du code de la Sécurité sociale) peut être accordée en cas :

- D'affection grave, caractérisée, de forme évolutive ou invalidante et reconnue par le corps médical, nécessitant des soins continus d'une durée prévisible supérieure à six mois et une thérapeutique coûteuse. Typiquement, les cancers sont concernés puisque les traitements sont longs (généralement plus de six mois) et coûteux ;

- De polypathologie entraînant un état pathologique invalidant, nécessitant des soins continus d'une durée prévisible supérieure à six mois.

Depuis la loi du 13 août 2004, la reconnaissance d'une ALD est soumise à la rédaction d'un protocole de soins, réalisé par le médecin traitant, définissant le parcours de soins du patient (les soins et les traitements nécessaires à la prise en charge de la maladie). Ce protocole se compose de trois volets; le premier est conservé par le médecin traitant, le second est destiné au médecin conseil de la Caisse primaire d'assurance maladie (CPAM) qui donne son accord pour la prise en charge à $100 \%$ d'une partie ou de la totalité des soins et des traitements liés à la maladie. Enfin, le troisième volet personnel et confidentiel est destiné au patient. Le protocole de soins est rédigé par le médecin traitant en charge du suivi du patient et au titre de la coordination des soins. Les soins associés directement à une ALD sont pris en charge à $100 \%$.

L'exonération initiale est accordée pour une durée de cinq ans, renouvelable dès lors que la poursuite d'une thérapeutique ou la prise en charge diagnostique et thérapeutique des séquelles liées à la maladie ou aux traitements sont nécessaires. Par ailleurs, toute récidive ou apparition d'une séquelle tardive grave, dont le lien de causalité avec le traitement est établi, conduit à la reprise de l'exonération du ticket modérateur (article annexe à l'article D322-1 du code de la Sécurité sociale).

Par ailleurs, la Cnamts évalue annuellement les coûts liés à la prise en charge des ALD. À titre d'exemple, le projet de loi de financement de la sécurité sociale en 2015 reporte que le coût moyen annuel de prise en charge d'un cancer en 2012 est de $4759 €$, mais il s'élève à $10179 €$ l'année pour les patients en phase active contre $1312 €$ pour les patients en phase de surveillance. 
Encadré 3

\section{LA MÉTHODE DE DIFFÉRENCE DES DIFFÉRENCES AVEC APPARIEMENT EXACT}

La méthode de différence des différences avec appariement exact appartient à l'ensemble des méthodes d'évaluation d'impact (Fougère, 2010). Son but est d'évaluer l'effet moyen sur un résultat d'un « traitement » dont bénéficie (ou est victime) une partie de la population considérée. Dans cette étude, le traitement considéré est la survenue du premier cancer enregistré en ALD cancer, tandis que les variables de résultat sont des situations sur le marché du travail (arrêt maladie, chômage ou emploi) et des durées trimestrielles passées dans chaque situation. Notre analyse porte donc sur les probabilités d'être dans ces trois situations.

Nous noterons ici $p_{i, t}$ la probabilité d'être dans une situation particulière, par exemple l'emploi, le modèle se présentant de manière identique quelle que soit la variable de résultat considérée. Chaque individu $i$ est observé entre les dates $t_{i}^{-}$et $t_{i}^{+}$, tandis que la survenue du cancer, le traitement, a lieu à la date $t_{i} \in\left[t_{i}^{-}, t_{i}^{+}\right]$. Afin d'évaluer l'effet de la survenue du cancer, on compare donc la situation professionnelle de la date située immédiatement avant la survenue du cancer $t_{i}-1$, à la situation professionnelle de la date située $k$ années après la survenue du cancer $t_{i}+k, t_{i}+k \leq t_{i}^{+}$.

L'indicatrice d'emploi pour l'individu $i$, notée $d_{i t}$, suit un processus de Bernoulli de probabilité conditionnelle $p_{i t}$, ce que l'on peut représenter par le modèle suivant :

$d_{i t}=p_{i t}+\varepsilon_{i t}$

$p_{i t}=f\left(X_{i}\right)+\alpha_{i}+\beta_{t}\left(X_{i}\right)+\gamma_{i} \cdot T_{i t}$

avec $i=1, \ldots, N, t \in\left[t_{i}^{-}, t_{i}^{+}\right]$et $E\left(\varepsilon_{i t} \mid X_{i}, \alpha_{i}, \beta_{t}\left(X_{i}\right), T_{i t}\right)=0$.

Dans ce modèle $f\left(X_{i}\right)$ représente l'effet des variables individuelles observables, $\alpha_{i}$ l'effet des variables individuelles inobservables, $\beta_{t}\left(X_{i}\right)=\beta_{0 t}+\beta_{1 t}\left(X_{i}\right)$ l'effet des variables temporelles inobservables que l'on décompose en deux parties : $\beta_{0 t}$ est l'effet temporel commun à tous les individus (comme par exemple les effets de la conjoncture sur le marché du travail) et $\beta_{1 t}\left(X_{i}\right)$ l'effet temporel spécifique aux individus de caractéristiques observables $X_{i}$ (effet dit "croisé "). $T_{i t}$ est une variable indicatrice du traitement qui vaut 1 si l'individu $i$ est atteint par le cancer et $t \geq t$, et qui vaut 0 si l'individu traité n'est pas encore atteint de cancer $\left(t<t_{j}\right) \cdot \varepsilon_{i t}$ est un terme d'erreur idiosyncratique non corrélé avec les autres effets, dont l'espérance est constante sans perte de généralité. Le paramètre $\gamma_{i}$ est le paramètre d'intérêt puisqu'il mesure l'effet du traitement sur la probabilité d'être en emploi pour l'individu $i$.

Nous utilisons une généralisation de la modélisation classique sur données de panel. La méthode d'appariement permet de ne pas avoir à spécifier la fonction $f\left(X_{i}\right)$ qui relie les variables observables aux variables de résultat. Pour l'hétérogénéité inobservée, la modélisation est exactement la même que dans un modèle de données de panel classique. On prend la différence des différences pour éliminer l'hétérogénéité inobservée (constante par individu ou dans le temps). C'est la méthode d'estimation qui change. Avec une méthode classique on doit spécifier $f(X)$ et estimer tous les coefficients. Ceci pose le problème du choix de la forme fonctionnelle et éventuellement des problèmes de multicolinéarité approchée (impossibles avec une méthode d'appariement). Enfin, notre panel n'est pas cylindré, ce qui ne cause aucune gêne quand on apparie. Les gains pratiques sont importants pour l'estimation. II y a donc un gain de généralité, on ne spécifie pas la fonction $f(X)$, et un grain pratique, on estime plus facilement qu'avec des moindres carrés sur données en différences.

L'estimation de la valeur moyenne de $\gamma_{i}$ peut être décomposée en trois étapes :

- La première étape permet de comparer la situation professionnelle entre les individus ayant expérimenté la survenue d'un cancer avec les individus qui n'ont fait l'objet d'aucun enregistrement pour une affection chronique de longue durée, cancer ou autres.

La situation professionnelle d'un individu $i$ victime d'un cancer est, une année avant sa survenue, donnée par $d_{i t_{i}-1}=f\left(X_{i}\right)+\alpha_{i}+\beta_{t_{i}-1}\left(X_{i}\right)+\varepsilon_{i t_{i}-1}$. Celle d'un individu $j$ qui ne connaîtra pas de cancer ou d'autres maladies reconnues en Affection de longue durée (ALD) est, à la même date, égale à :

$d_{j t_{i}-1}=f\left(X_{j}\right)+\alpha_{j}+\beta_{t_{i}-1}\left(X_{j}\right)+\varepsilon_{j t_{i}-1}$.

Dans un premier temps, nous allons apparier les deux catégories d'individus précédents selon la valeur exacte des variables explicatives observables. Cela signifie que pour tout individu $i$, on recherche les individus $j$ qui ont les mêmes valeurs pour les variables explicatives observables $X$, c'est-à-dire tels que: $j \in J(i)=\left\{j \mid X_{i}=X_{j}\right\}$. L'ensemble $J(i)$ est l'ensemble de tous les jumeaux de l'individu traité $i$. II est important de noter que pour chaque individu avec un cancer, individu dit « traité " $i$, on peut trouver plusieurs individus non-traités avec les mêmes valeurs des variables explicatives. Chaque individu traité possède donc éventuellement plusieurs individus jumeaux. De même, chaque individu non atteint de cancer peut servir de jumeau pour plusieurs individus avec un cancer si là encore ils partagent la même valeur des variables observables. Nous utilisons tous les jumeaux de chaque individu, avec remise. Un jumeau peut donc servir plusieurs fois.

Parce que nous avons apparié les individus qui n'ont pas subi de cancer selon les périodes de maladie des individus qui ont été malades du cancer, la comparaison de leur situation avant la maladie est réalisée à la même date $t_{i}-1$. L'appariement porte donc aussi implicitement sur les dates de présence des individus traités et témoins.

Nous calculons tout d'abord la différence, avant la survenue du cancer, entre la situation professionnelle d'un individu traité $i$ et le résultat moyen de tous les 
prédispositions génétiques au développement de certains types de cancers, car on compare deux résultats du même individu. La même différence est réalisée dans la population des individus qui n'ont pas eu de cancer en prenant comme date de comparaison la date de survenue du cancer de leur jumeau malade.

La troisième étape consiste à effectuer la différence des différences des résultats des deux individus comparés. Le fait que les individus soient suivis sur la même période permet à cette double différence de neutraliser les effets temporels inobservés.

Enfin, on calcule la moyenne de ces effets sur l'ensemble des individus de l'échantillon.
La méthode de différences des différences peut être appréciée comme une méthode non paramétrique au sens où on ne spécifie pas de forme fonctionnelle entre les variables expliquées et explicatives, ou de distribution des termes d'erreur. Il y a bien une hypothèse d'additivité des effets individuels observables, non observables et temporels, mais elle est affaiblie par l'autorisation d'effets temporels différents selon la valeur des variables d'appariement (effets croisés individuels-temporels). Globalement, nos estimations ne font que des hypothèses très faibles de (semi) additivité et aucune hypothèse de distribution, de sorte qu'on peut considérer qu'elles font partie de la littérature non paramétrique. Cette méthode a été choisie afin de renforcer la robustesse des résultats obtenus dans

Encadré 3 (suite)

individus témoins appariés. Soit $N(i)=\operatorname{Card}(J(i))$ le nombre de jumeaux du traité $i$. On obtient :

$$
\begin{aligned}
D_{t_{i}-1} & =d_{i t_{i}-1}-\frac{1}{N(i)} \sum_{j \in J(i)} d_{j t_{i}-1} \\
& =\alpha_{i}-\alpha_{j}+\varepsilon_{i t_{i}-1}-\frac{1}{N(i)} \sum_{j \in J(i)} \varepsilon_{j t_{i}-1}
\end{aligned}
$$

La deuxième étape revient à réaliser la même différence après la survenue du cancer, c'est-à-dire à un horizon $t_{i}+k$ choisi. II est possible de réaliser cette différence une année après la survenue du cancer ou $k$ années après. Dans cette étude, nous faisons varier l'horizon d'observation des conséquences du cancer sur la situation professionnelle entre $k=1$ et $k=5$ années.

$k$ années après la survenue du cancer, la différence de situation professionnelle d'un individu traité $i$ et d'individu témoin apparié $j$ est donc :

$$
\begin{aligned}
D_{t_{i}+k} & =d_{i t_{i}+k}-\frac{1}{N(i)} \sum_{j \in J(i)} d_{j t_{i}+k} \\
& =\gamma_{i}+\alpha_{i}-\alpha_{j}+\varepsilon_{i t_{i}+k}-\frac{1}{N(i)} \sum_{j \in J(i)} \varepsilon_{j t_{i}+k}
\end{aligned}
$$

Par rapport à la différence (2) calculée avant la maladie, l'expression (3) contient l'effet du cancer sur la situation professionnelle de l'individu traité. En revanche, l'effet temporel inobservable $\left(\beta_{0 t}\right)$ a été éliminé dans l'expression (3) comme dans l'expression (2) puisque les situations professionnelles de l'individu traité et de l'individu témoin sont observées à une période commune fixée par le temps passé depuis le cancer de l'individu dit « traité ». De même, l'effet temporel croisé $\left(\beta_{1 t}(X)\right)$ a été éliminé grâce à la conjonction de l'appariement sur les variables individuelles observables et de la période d'appariement.
La dernière étape consiste à faire la différence des différences de résultat calculées ci-dessus (3)-(2) :

$$
\begin{array}{r}
D_{t_{i}+k}-D_{t_{i}-1}=\gamma_{i}+ \\
\varepsilon_{i t_{i}+k}-\frac{1}{N(i)} \sum_{j \in J(i)} \varepsilon_{j t_{i}+k} \\
-\varepsilon_{i t_{i}-1}+\frac{1}{N(i)} \sum_{j \in J(i)} \varepsilon_{j t_{i}-1}
\end{array}
$$

Sous l'hypothèse que l'espérance du terme d'erreur idiosyncratique est variable selon la date, mais égale pour tous les individus à une date donnée, c'est-à-dire que $E\left(\varepsilon_{j t}\right)=\mu_{t}$, l'espérance de la double différence (4) se réduit à l'effet pour l'individu $i$ de la survenue du cancer sur la situation professionnelle $k$ années après celle-ci.

Nous devons estimer la valeur moyenne des effets du cancer sur l'ensemble des individus ayant été atteints du cancer (effet moyen du traitement sur les traités), de sorte que nous prenons l'estimateur suivant :

$\hat{\gamma}=\frac{1}{N_{h}} \sum_{i=1}^{N_{h}}\left(D_{t_{i}+k}-D_{t_{i}-1}\right)$

où $N_{h}$ est le nombre d'individus appariables pour lesquels le cancer est survenu. Le taux d'appariement est égal à $N_{h} / N$, où $N$ est le nombre d'individus traités.

II reste à estimer les écarts types des quantités estimées. Pour cela, nous employons la méthode du bootstrap sur 500 tirages. Le tirage des individus se fait globalement ("par grappe ") sur les bases contenant les individus ayant ou non connu un cancer. Le bootstrap tient donc compte de la variation du nombre d'individus appariables.

Toutes les estimations ont été réalisées sous SAS 9.4 par les auteurs. 
le contexte relativement difficile de l'évaluation à partir d'un modèle dynamique sur panel non cylindré.

Les paramètres estimés mesurent le changement de situation professionnelle vécu par les salariés après la survenue du cancer, en termes de fréquence et de durée d'emploi, de chômage et d'arrêt maladie. Le fait d'apparier les individus sur des périodes communes d'observation permet de faire varier la durée de la fenêtre de comparaison et de mesurer précisément les effets de la survenue de la maladie sur le court (0 à 1 an) ou le moyen terme ( 2 à 5 ans). De plus, les effets sur la situation professionnelle sont estimés selon le genre et l'âge des malades lors de la survenue du cancer.

Malgré les précautions que nous avons prises, certaines variables inobservables, non constantes dans le temps, pourraient influencer à la fois les parcours professionnels et la santé. Pour réduire les biais hypothétiques d'évaluation qui pourraient survenir suite à leur omission, nous avons choisi d'intégrer, parmi les variables d'appariement, des indicateurs retardés de situation professionnelle datés de l'année précédant la survenue du cancer.

\section{Les variables}

Les variables de résultat:

les mesures de la situation professionnelle

Les variables de résultat concernent la situation et la durée sur le marché du travail. La base Hygie fournit des informations sur l'emploi, les arrêts de travail (maladie, maternité, accident du travail) et les épisodes de chômage indemnisés sur l'ensemble de la carrière. Ce premier type de variables permet de calculer une probabilité d'être dans une situation professionnelle donnée à une date spécifique de la carrière.

Les situations professionnelles sont identifiées dans la base Hygie à travers le nombre annuel de trimestres cotisés en tant que salarié dans le Régime général et le nombre annuel de trimestres correspondant à des périodes assimilées - maladie, maternité, accident du travail, chômage-dans ce même régime. Les trimestres cotisés en situation d'emploi salarié ne permettent pas de mesurer précisément la durée d'emploi sur l'année parce que la validation d'un trimestre d'emploi est associée à un niveau de salaire. En effet, le versement d'un salaire équivalent à 200 heures de Smic valide un trimestre, ce qui signifie que le titulaire d'un salaire élevé, situé au-dessus du plafond de la Sécurité sociale, peut valider un trimestre en travaillant moins de trois mois.

De même, les trimestres validés en maladie ${ }^{11}$ ou au chômage ne permettent pas non plus de calculer la durée précise associée à ces situations. Ainsi, une période est assimilée à un trimestre d'assurance maladie chaque fois que l'assuré a bénéficié d'au moins 60 jours consécutifs d'indemnisation au titre de l'Assurance maladie. De plus, un salarié valide un trimestre en tant que période assimilée au titre du chômage lorsqu'il réunit au moins 50 jours de chômage indemnisé consécutifs, dans la limite de quatre trimestres par année civile.

Pour tenir compte de ces difficultés de mesure temporelle, nous allons identifier l'occurrence dans chaque situation (chômage, maladie et emploi) à la suite de la validation d'un trimestre ou plus dans une situation pour chaque année. À partir des trimestres validés en emploi, chômage et maladie, on utilise finalement les deux variables suivantes pour chacune des trois situations (emploi, maladie, chômage) :

1. La situation: avoir validé un trimestre ou non pour chacune des trois situations, emploi, chômage et maladie. Pour le même individu, plusieurs indicatrices peuvent êtres égales à 1 simultanément ;

2. La durée : le nombre total de trimestres validés dans une des trois situations (emploi, maladie, chômage).

Chacun de ces indicateurs présente des particularités. La variable d'indicatrice de situation professionnelle enregistre a minima le passage par l'une de ces trois situations au cours d'une année. Elle ne permet donc pas de mesurer l'importance relative du temps passé dans ces situations dans les années suivant la déclaration du cancer. D'autre part, pour le nombre de trimestres validés, la relation imparfaite entre le temps effectif passé dans une situation et l'enregistrement administratif des trimestres conduit à des imperfections de mesure de la durée, en particulier dans le cas des trimestres en emploi, dont la variation peut être d'autant plus importante que le

11. Nous parlons ici de situation de maladie à fin de simplification pour qualifier de manière étendue une situation professionnelle d'arrêt de travail qui couvre également les accidents du travail et la maternité. 
salarié perçoit un salaire élevé par rapport au plafond de la Sécurité sociale.

Du fait de leur complémentarité, nous présentons les estimations sur ces deux types d'indicateurs (situation, durée).

\section{Les variables d'appariement}

Les variables utilisées pour l'appariement sont d'abord la date de première inscription en ALD cancer (puisqu'on apparie par date de la survenue du cancer via la première inscription en $\mathrm{ALD})^{12}$, le genre, l'année de naissance et la classe de salaire à l'entrée sur le marché du travail, c'est-à-dire après cotisation de quatre trimestres en activité. Cette dernière variable représente une mesure indirecte de la catégorie socioprofessionnelle et du niveau d'éducation du salarié en début de carrière. Afin de se prémunir de potentiels biais de sélection, nous apparions aussi sur la situation sur le marché du travail l'année précédant la survenue du cancer (endogène retardée). Spécifiquement, ce sont les trois situations retardées d'emploi, de chômage et de maladie qui sont utilisées. À ce titre, il convient de noter que les individus entrant en ALD à une date $t$ peuvent l'année précédente être caractérisés par une forme d'inactivité, correspondant à une situation où ils n'ont validé aucun trimestre dans les trois situations d'emploi, de chômage et de maladie. Dans ce cas, l'appariement leur associe un individu sans ALD également inactif à cette date.

\section{Résultats}

\section{Statistiques générales}

Le tableau 1 décrit la distribution des différents types de cancer selon le sexe du malade.

12. La date de première inscription en ALD Cancer est une variable d'appariement mais la survenue du cancer est la variable de traitement. Ainsi, cette date de première inscription est l'année de référence pour la population atteinte par le cancer. De ce fait, notre échantillon intègre des individus dont le cancer est survenu à des dates très différentes, avec pour certains d'entre eux une ancienneté très importante.

Tableau 1

Types de cancer de fréquence supérieure à $2 \%$ (Regroupement des deux principaux codes de maladie de chaque individu)

\begin{tabular}{|c|c|c|}
\hline CIM-10 & Libellé & Pourcentage \\
\hline \multicolumn{3}{|l|}{ Femmes } \\
\hline C50 & Tumeur maligne du sein & 47,4 \\
\hline C73 & Tumeur maligne de la thyroïde & 6,5 \\
\hline C53 & Tumeur maligne du col de l'utérus & 3,7 \\
\hline C18 & Cancer du côlon & 2,9 \\
\hline C56 & Tumeur maligne de l'ovaire & 2,7 \\
\hline C43 & Mélanome malin de la peau & 2,6 \\
\hline C54 & Tumeur maligne du corps de l'utérus & 2,2 \\
\hline C34 & Tumeur maligne des bronches et du poumon & 2,0 \\
\hline \multicolumn{3}{|l|}{ Hommes } \\
\hline C61 & Tumeur maligne de la prostate & 13,9 \\
\hline C34 & Tumeur maligne des bronches et du poumon & 9,0 \\
\hline C62 & Tumeur maligne du testicule & 4,8 \\
\hline C67 & Tumeur maligne de la vessie & 4,6 \\
\hline C18 & Cancer du côlon & 4,4 \\
\hline C64 & Tumeur maligne du rein, à l'exception du bassinet & 2,9 \\
\hline C32 & Tumeur maligne du larynx & 2,8 \\
\hline $\mathrm{C} 73$ & Tumeur maligne de la thyroïde & 2,5 \\
\hline C20 & Tumeur maligne du rectum & 2,4 \\
\hline C43 & Mélanome malin de la peau & 2,3 \\
\hline
\end{tabular}

Lecture : le tableau reporte la fréquence des types de cancer dans la base Hygie pour les cancers de fréquence supérieure à $2 \%$ Le codage des cancers utilisé est effectué selon la Classification internationale des maladies, $10^{\circ}$ édition, CIM-10.

Champ : tout l'échantillon.

Source : Hygie (Irdes). 
Il montre que les tumeurs malignes du sein pour les femmes représentent $47 \%$ des déclarations en ALD cancer. Pour les hommes, les tumeurs malignes de la prostate sont les plus prévalentes et représentent $14 \%$ tandis que les tumeurs malignes des bronches et du poumon représentent $9 \%$ de l'ensemble des ALD.

Dans notre base, l'âge médian pour un premier enregistrement en ALD cancer est de 48 ans pour les femmes et de 51 ans pour les hommes, soit des âges médians nettement plus jeunes que ceux constatés selon les sources épidémiologiques de recensement de l'âge médian de l'incidence des cancers (voir Inca, 2015) ${ }^{13}$. Cet écart d'âge médian est lié à la structure de notre échantillon qui est un échantillon relativement « jeune » du fait de la restriction imposée par la
Cnav de ne sélectionner que des individus d'un âge inférieur à 70 ans, alors même que le cancer, en dehors des cancers des enfants, est une maladie du vieillissement. Cette caractéristique propre à notre base permet d'évaluer l'effet du cancer sur les parcours professionnels pour un échantillon très important d'individus relativement jeunes, ce que peu de bases permettent en France.

Le tableau 2 compare les variables d'appariement des individus sans aucune ALD avec

13. Pour le cancer de la prostate, l'âge médian au diagnostic en 2009 est un peu avant 70 ans; pour le côlon, l'âge médian au diagnostic en 2012 est de 71 ans chez l'homme, 75 ans chez la femme; pour le poumon en 2012, il est de 66 ans chez l'homme et 65 ans chez la femme. Enfin pour le sein, cancer quasi exclusivement féminin, il est de 63 ans en 2012 (voir Inca, 2015).

Tableau 2

Comparaison des individus sans ALD et avec ALD cancer, selon les variables d'appariement

\begin{tabular}{|c|c|c|c|}
\hline & $\begin{array}{c}\text { Aucune ALD } \\
\text { (1) }\end{array}$ & $\begin{array}{c}\text { ALD cancer } \\
\text { (2) }\end{array}$ & $\begin{array}{l}\text { Écart } \\
(2)-(1)\end{array}$ \\
\hline \multicolumn{4}{|l|}{ Hommes } \\
\hline Fréquence & 219447 & 4739 & \\
\hline \multicolumn{4}{|c|}{ Classes d’âge (en 2008) } \\
\hline [25 - 52] & 72,3 & 28,1 & $-44,2$ \\
\hline$[53-58]$ & 9,8 & 22,1 & 12,3 \\
\hline$[59-63]$ & 7,5 & 25,7 & 18,2 \\
\hline$[64-73]$ & 10,4 & 24,1 & 13,8 \\
\hline \multicolumn{4}{|c|}{ Classes de salaires annuels d'entrée sur le marché du travail (en euros constants 2005) } \\
\hline Moins de 2801 & 12,1 & 19,2 & 7,0 \\
\hline [2 802 - 8 694] & 16,8 & 35,6 & 18,8 \\
\hline [8 695 - 14 711] & 18,9 & 21,0 & 2,2 \\
\hline [14 712 - 20 383] & 24,1 & 15,3 & $-8,8$ \\
\hline Plus de 20384 & 28,1 & 8,9 & $-19,1$ \\
\hline \multicolumn{4}{|l|}{ Femmes } \\
\hline Fréquence & 203491 & 5761 & \\
\hline \multicolumn{4}{|c|}{ Classes d'âge (en 2008) } \\
\hline$[25-52]$ & 74,3 & 38,5 & $-35,9$ \\
\hline$[53-58]$ & 10,0 & 23,8 & 13,8 \\
\hline$[59-63]$ & 6,7 & 20,5 & 13,8 \\
\hline$[64-73]$ & 9,0 & 17,2 & 8,2 \\
\hline \multicolumn{4}{|c|}{ Classes de salaires annuels d'entrée sur le marché du travail (en euros constants de 2005) } \\
\hline Moins de 2801 & 22,7 & 32,1 & 9,4 \\
\hline [2 802 - 8694$]$ & 19,7 & 31,1 & 11,4 \\
\hline [8 695 - 14 711] & 21,8 & 20,8 & $-1,0$ \\
\hline [14 712 - 20 383] & 19,0 & 10,8 & $-8,2$ \\
\hline Plus de 20384 & 16,8 & 5,1 & $-11,7$ \\
\hline
\end{tabular}

Lecture : pour chaque genre, le tableau reporte les pourcentages d'individus selon les variables d'appariement, pour l'ensemble de ceux enregistrés en ALD cancer et de ceux enregistrés dans aucune ALD. La cinquième ligne du tableau renseigne sur le fait que $72,3 \%$ des individus sans ALD et $28,1 \%$ des individus avec ALD cancer sont âgés de 25 à 52 ans.

Champ : tout l'échantillon.

Source : Hygie (Irdes). 
celles des individus enregistrés en ALD cancer. Pour les hommes comme pour les femmes, les individus atteints d'un cancer sont en 2008 nettement plus âgés que les individus sans aucune ALD. Parmi les hommes enregistrés en ALD cancer, $72 \%$ ont plus de 52 ans alors que cette classe d'âge ne représente que $28 \%$ des hommes sans ALD. Les femmes atteintes de cancer sont un peu plus jeunes ; $62 \%$ d'entre elles sont âgées de plus de 52 ans contre $26 \%$ des femmes sans ALD. Ces observations confirment que le cancer est d'abord une maladie qui touche les populations plus âgées, en particulier chez les hommes.

Par ailleurs, nous avons calculé les pourcentages d'individus avec ALD cancer et sans aucune ALD dans les quintiles de la distribution des salaires d'entrée sur le marché du travail, en euros constants 2005, pour l'ensemble des individus. Les individus atteints d'un cancer disposent d'un salaire d'entrée sur le marché du travail plus faible que les individus sans ALD. Ainsi $55 \%$ des hommes et $63 \%$ des femmes atteints d'un cancer appartiennent aux deux premiers quintiles de la distribution contre $29 \%$ des hommes et $42 \%$ des femmes sans ALD. Cette différence brute démontre la trace d'une inégalité sociale de santé, établissant un lien entre l'appartenance à une classe de revenus salariaux en début de carrière et la survenue du cancer. Nous ne pouvons pas ici différencier les origines de cette inégalité (antécédents de santé non reconnus en ALD, comportements à risque, accès à la prévention, etc.), mais cette observation nous conduit à interroger la possibilité que les inégalités sur le marché du travail interfèrent avec les inégalités de santé.

Le tableau 3 compare les caractéristiques en termes de situation professionnelle en 2008 (dernière année renseignée dans la base Hygie) des individus sans aucune ALD et avec ALD cancer. Il permet ainsi de décrire les écarts bruts dans une perspective naïve sans recours à l'appariement.

Tableau 3

Comparaison des individus sans ALD et avec ALD cancer, selon les situations professionnelles

\begin{tabular}{|c|c|c|c|}
\hline & $\begin{array}{l}\text { Sans aucune ALD } \\
\text { (1) }\end{array}$ & $\begin{array}{c}\text { ALD cancer } \\
\text { (2) }\end{array}$ & $\begin{array}{l}\text { Écart } \\
\text { (2)-(1) }\end{array}$ \\
\hline \multicolumn{4}{|l|}{ Hommes } \\
\hline Nombre & 219447 & 4739 & \\
\hline \multicolumn{4}{|c|}{ Situation en 2008 (proportion) } \\
\hline Chômage & 0,171 & 0,216 & 0,045 \\
\hline Maladie & 0,030 & 0,201 & 0,172 \\
\hline Emploi & 0,810 & 0,551 & $-0,259$ \\
\hline \multicolumn{4}{|c|}{ Durée en 2008 (nombre de trimestres) } \\
\hline Chômage & 0,506 & 0,658 & 0,152 \\
\hline Maladie & 0,058 & 0,552 & 0,494 \\
\hline Emploi & 3,075 & 1,931 & $-1,143$ \\
\hline \multicolumn{4}{|l|}{ Femmes } \\
\hline Nombre & 203491 & 5761 & \\
\hline \multicolumn{4}{|c|}{ Situation en 2008 (proportion) } \\
\hline Chômage & 0,179 & 0,195 & 0,015 \\
\hline Maladie & 0,070 & 0,195 & 0,126 \\
\hline Emploi & 0,766 & 0,601 & $-0,165$ \\
\hline \multicolumn{4}{|c|}{ Durée en 2008 (nombre de trimestres) } \\
\hline Chômage & 0,527 & 0,596 & 0,069 \\
\hline Maladie & 0,110 & 0,533 & 0,423 \\
\hline Emploi & 2,838 & 2,123 & $-0,715$ \\
\hline
\end{tabular}

Lecture : pour chaque genre, le tableau reporte les moyennes des variables de résultat pour l'ensemble des individus enregistrés en ALD cancer et des individus enregistrés dans aucune ALD. La cinquième ligne du tableau renseigne sur le fait que les individus sans ALD ont enregistré au moins un trimestre de chômage pendant $17,1 \%$ des années de leur carrière, tandis que les individus avec ALD cancer ont passé au moins un trimestre au chômage pendant 21,6 \% des années de leur carrière. La neuvième ligne renseigne sur le fait que les individus sans ALD ont validé en moyenne 0,506 trimestre en chômage par an durant leur carrière, tandis que les individus en ALD cancer ont validé en moyenne 0,658 trimestre en chômage par an durant la leur.

Champ : tout l'échantillon.

Source : Hygie (Irdes). 
Respectivement $55 \%$ et $60 \%$ des hommes et femmes ayant souffert d'un cancer ont validé au moins un trimestre d'emploi durant cette année. Cette proportion est beaucoup plus forte chez les personnes en bonne santé : $81 \%$ pour les hommes et $77 \%$ pour les femmes. Par ailleurs, le nombre de trimestres en emploi des hommes et des femmes avec ALD diminue respectivement de 1,93 et 2,12 trimestres. De plus, les personnes souffrant de cancer sont plus souvent sujettes aux arrêts maladie : $20 \%$ d'entre elles ont validé au moins un trimestre de maladie en 2008, contre respectivement $3 \%$ et $7 \%$ des hommes et femmes sans aucune ALD. Les individus souffrant d'un cancer ont validé en moyenne un peu plus d'un demi-trimestre de maladie en 2008 contre environ 0,1 trimestre dans la population sans ALD.

Il est également notable que les personnes en ALD ont plus fréquemment validé de trimestres au titre du chômage durant l'année 2008.
Le tableau 4 livre des informations sur les taux d'appariement et la qualité de l'appariement. Les taux d'appariement sont de l'ordre de $99 \%$.

\section{La situation professionnelle post-cancer selon le genre}

Nous présentons les effets du cancer sur la situation professionnelle selon trois caractéristiques des individus. Tout d'abord, les tableaux $5 \mathrm{~A}$ et $5 \mathrm{~B}$ examinent les différences globales de résultat professionnel sur le marché du travail selon le genre. Les tableaux 6 et 7 permettent d'apprécier ces différences en tenant compte de l'âge d'apparition du premier cancer enregistré en ALD (en distinguant deux sous-populations selon l'âge médian de survenue du cancer). Enfin les graphiques I et II soulignent les différences de résultats à la suite d'un cancer selon les quintiles de la distribution des salaires d'entrée sur le marché du travail et ce afin de rendre

Tableau 4

Qualité des appariements

\begin{tabular}{|c|c|c|}
\hline Femmes & $t+1$ & $t+5$ \\
\hline (1) Traitées & 5537 & 3063 \\
\hline (2) Non traitées : variables individuelles identiques & 5525 & 3057 \\
\hline (1)-(2) Perte & 12 & 6 \\
\hline (3) Non traitées : périodes de présence compatibles & 5518 & 3057 \\
\hline (2)-(3) Perte & 7 & 0 \\
\hline (4) Non traitées : variables retardées identiques & 5488 & 3042 \\
\hline (3)-(4) Perte & 30 & 15 \\
\hline (4)/(1) Taux d'appariement & $99,1 \%$ & $99,3 \%$ \\
\hline Nombre moyen de jumelles par traitée appariable & 597,6 & 607,5 \\
\hline Hommes & $t+1$ & $t+5$ \\
\hline (1) Traités & 4625 & 2191 \\
\hline (2) Non traités : variables individuelles identiques & 4624 & 2191 \\
\hline (1)-(2) Perte & 1 & 0 \\
\hline (3) Non traités : périodes de présence compatibles & 4616 & 2187 \\
\hline (2)-(3) Perte & 8 & 4 \\
\hline (4) Non traitées : variables retardées identiques & 4564 & 2166 \\
\hline (3)-(4) Perte & 52 & 21 \\
\hline (4)/(1) Taux d'appariement & $98,7 \%$ & $98,9 \%$ \\
\hline Nombre moyen de jumeaux par traité appariable & 959,5 & 1065,4 \\
\hline
\end{tabular}

Note : les appariements sont d'abord réalisés selon les variables individuelles, puis on vérifie la compatibilité des périodes de présence pour les individus appariables. Enfin, on vérifie que les variables retardées sont identiques.

Lecture : si on évalue l'effet du cancer à un an, on dispose d'un échantillon de 5537 femmes. Seulement 12 de ces femmes n'ont pas de jumelle au sens des variables individuelles parmi les femmes qui n'ont pas eu de cancer. Parmi les 5525 femmes « traitées » restantes, 5518 ont au moins une jumelle présente sur une période recouvrant leur période de présence. Parmi ces 5518 " traitées » restantes, 5488 ont au moins une jumelle qui était dans la même situation d'activité un an avant l'apparition de leur cancer. Globalement, le taux d'appariement est de 99,1\%. Parmi ces 5488 traitées appariables, le nombre moyen de jumelles disponibles est de 597,6 par traitée (avec remise).

Champ : tout l'échantillon

Source : Hygie (Irdes). 
Tableau 5A

Effet du cancer sur l'activité professionnelle, selon le genre

\begin{tabular}{|c|c|c|c|c|c|c|c|}
\hline & \multirow{2}{*}{$\begin{array}{c}\text { Nombre de traités } \\
\text { Taux d'appariement (en \%) }\end{array}$} & \multicolumn{3}{|c|}{ Situation (en \%) } & \multicolumn{3}{|c|}{ Durée (en trimestres) } \\
\hline & & Emploi & Maladie & Chômage & Emploi & Maladie & Chômage \\
\hline \multicolumn{8}{|l|}{ Femmes } \\
\hline \multicolumn{8}{|l|}{$t-1, t+1$} \\
\hline$E\left(y_{0} \mid T=1\right)$ & 5537 & 95,9 & 0,6 & 3,4 & 3,73 & 0,01 & 0,11 \\
\hline Effet & 99,0 & $-8,2^{\star \star}$ & $49,9^{* *}$ & $-2,0^{\star *}$ & $-0,51^{* *}$ & $1,68^{\star \star}$ & $-0,07^{\star \star}$ \\
\hline Student & & 18,53 & 78,53 & 6,59 & 26,66 & 70,92 & 6,89 \\
\hline \multicolumn{8}{|l|}{$t-1, t+2$} \\
\hline$E\left(y_{0} \mid T=1\right)$ & 4836 & 96,3 & 0,6 & 3,0 & 3,74 & 0,01 & 0,10 \\
\hline Effet & 99,0 & $-7,6^{\star \star}$ & $27,5^{\star \star}$ & $-0,3$ & $-0,40^{\star \star}$ & $0,92^{\star \star}$ & $-0,02$ \\
\hline Student & & 17,03 & 43,66 & 0,84 & 20,63 & 42,40 & 1,25 \\
\hline \multicolumn{8}{|l|}{$t-1, t+3$} \\
\hline$E\left(y_{0} \mid T=1\right)$ & 4211 & 96,4 & 0,6 & 2,7 & 3,75 & 0,01 & 0,09 \\
\hline Effet & 99,2 & $-9,0^{\star *}$ & $16,3^{* *}$ & $0,9^{*}$ & $-0,42^{\star \star}$ & $0,45^{\star \star}$ & 0,03 \\
\hline Student & & 17,12 & 28,57 & 1,65 & 19,46 & 26,65 & 1,54 \\
\hline \multicolumn{8}{|l|}{$t-1, t+4$} \\
\hline$E\left(y_{0} \mid T=1\right)$ & 3581 & 96,4 & 0,6 & 2,5 & 3,75 & 0,01 & 0,08 \\
\hline Effet & 99,3 & $-11,0^{\star \star}$ & $6,3^{\star *}$ & $1,2^{*}$ & $-0,47^{\star \star}$ & $0,17^{\star \star}$ & $0,05^{\star \star}$ \\
\hline Student & & 17,80 & 12,16 & 1,90 & 18,43 & 12,42 & 2,44 \\
\hline \multicolumn{8}{|l|}{$t-1, t+5$} \\
\hline$E\left(y_{0} \mid T=1\right)$ & 3063 & 96,2 & 0,6 & 2,4 & 3,74 & 0,01 & 0,08 \\
\hline Effet & 99,3 & $-12,8^{\star \star}$ & $5,6^{\star \star}$ & 0,4 & $-0,54^{\star \star}$ & $0,17^{\star \star}$ & 0,04 \\
\hline Student & & 18,20 & 10,22 & 0,63 & 18,92 & 10,87 & 1,60 \\
\hline \multicolumn{8}{|l|}{ Hommes } \\
\hline \multicolumn{8}{|l|}{$t-1, t+1$} \\
\hline$E\left(y_{0} \mid T=1\right)$ & 4625 & 97,2 & 0,3 & 2,9 & 3,85 & 0,01 & 0,10 \\
\hline Effet & 98,4 & $-6,8^{\star \star}$ & $38,9^{\star \star}$ & 0,0 & $-0,45^{\star \star}$ & $1,24^{\star *}$ & $-0,01$ \\
\hline Student & & 15,28 & 53,57 & 0,21 & 22,98 & 48,47 & 0,65 \\
\hline \multicolumn{8}{|l|}{$t-1, t+2$} \\
\hline$E\left(y_{0} \mid T=1\right)$ & 3784 & 97,8 & 0,3 & 2,2 & 3,87 & 0,01 & 0,07 \\
\hline Effet & 98,4 & $-8,7^{\star *}$ & $23,4^{* *}$ & $1,1^{\star *}$ & $-0,45^{\star \star}$ & $0,77^{\star \star}$ & $0,05^{\star \star}$ \\
\hline Student & & 15,89 & 33,13 & 2,02 & 19,27 & 31,45 & 2,62 \\
\hline \multicolumn{8}{|l|}{$t-1, t+3$} \\
\hline$E\left(y_{0} \mid T=1\right)$ & 3157 & 98,1 & 0,2 & 1,8 & 3,88 & 0,00 & 0,06 \\
\hline Effet & 98,4 & $-9,8^{\star \star}$ & $15,7^{\star \star}$ & $2,5^{\star \star}$ & $-0,50^{\star *}$ & $0,44^{\star *}$ & $0,08^{\star \star}$ \\
\hline Student & & 14,18 & 21,63 & 4,04 & 17,74 & 19,62 & 3,65 \\
\hline \multicolumn{8}{|l|}{$t-1, t+4$} \\
\hline$E\left(y_{0} \mid T=1\right)$ & 2582 & 98,3 & 0,2 & 1,6 & 3,89 & 0,00 & 0,05 \\
\hline Effet & 98,4 & $-13,2^{\star \star}$ & $8,0^{\star *}$ & $2,7^{\star \star}$ & $-0,59^{\star \star}$ & $0,20^{\star \star}$ & $0,09^{\star \star}$ \\
\hline Student & & 17,11 & 12,43 & 4,18 & 18,81 & 11,58 & 3,93 \\
\hline \multicolumn{8}{|l|}{$t-1, t+5$} \\
\hline$E\left(y_{0} \mid T=1\right)$ & 2191 & 98,5 & 0,2 & 1,4 & 3,89 & 0,00 & 0,04 \\
\hline Effet & 98,4 & $-12,6^{\star \star}$ & $8,3^{\star \star}$ & $2,6^{\star *}$ & $-0,55^{\star \star}$ & $0,21^{\star \star}$ & $0,09^{\star \star}$ \\
\hline Student & & 14,75 & 12,04 & 3,56 & 16,04 & 11,10 & 3,41 \\
\hline
\end{tabular}

Note : effet d'un cancer sur l'activité. Différence des différences avec appariement exact. Les $t$ de Student sont estimés par le bootstrap par grappe sur 500 répétitions. Variables d'appariement : indicatrices d'emploi, de maladie et de chômage en t- 1 , année de naissance, genre, quintile de salaire en début de carrière. ${ }^{\star \star}$ : significatif au seuil de $5 \%$; ${ }^{*}$ : significatif au seuil de $10 \%$.

Lecture : pour chaque genre, le tableau mesure l'effet moyen du cancer sur les individus atteints entre une année avant la survenue de la maladie et $\mathrm{k}$ années après celle-ci, $\mathrm{k}=1, \ldots 5$. Les trois premières lignes de la colonne Emploi renseignent respectivement sur le fait que 95,9\% des femmes avec un cancer étaient en emploi au moins un trimestre un an avant le cancer, que l'effet de la survenue du cancer est de - 8,2 points de pourcentage, significativement différent de 0 pour une statistique de Student de 18,53.

Champ : tout l'échantillon.

Source : Hygie (Irdes). 
compte de phénomènes d'inégalités sociales de parcours professionnels (seuls les résultats pour les variables d'emploi et de maladie sont produits). L'encadré 4 ci-dessous décrit comment lire les résultats des régressions réalisées.

\section{L'emploi à court terme}

Nous observons dans le tableau 5A la situation professionnelle consécutive à l'apparition d'un cancer à court et moyen termes (de un à cinq ans). Considérant l'effet immédiat à un an, nos résultats montrent une forte diminution du pourcentage d'individus employés durant au moins un trimestre parmi les personnes atteintes d'un cancer. La proportion de personnes en situation d'emploi (validation d'au moins un trimestre en emploi) a diminué de 8,2 points de pourcentage (pp) passant de $95,9 \%$ à $87,7 \%$ pour les femmes et de 6,8 pp pour les hommes passant de $97,2 \%$ à $90,4 \%$. Le tableau 5B montre que l'effet négatif mesuré dans le court terme sur la probabilité comme la durée d'emploi un an après la survenue du cancer est significativement plus élevé pour les femmes que pour les hommes.

Nous pouvons apprécier l'effet de l'âge de primo-enregistrement sur la trajectoire en scindant nos échantillons en 4 sous-groupes au regard des âges médians ( $c f$. tableaux 6 et 7 ) : les femmes «jeunes » (moins de 48 ans), les femmes « âgées » (48 ans et plus), les hommes

Tableau 5B

Comparaison des effets entre les femmes et les hommes

\begin{tabular}{|c|c|c|c|c|c|c|}
\hline & \multicolumn{3}{|c|}{ Situation (en \%) } & \multicolumn{3}{|c|}{ Durée (en trimestres) } \\
\hline & Emploi & Maladie & Chômage & Emploi & Maladie & Chômage \\
\hline \multicolumn{7}{|l|}{$t+1$} \\
\hline Femmes & $-8,2$ & 49,9 & $-2,0$ & $-0,51$ & 1,68 & $-0,07$ \\
\hline Hommes & $-6,8$ & 38,9 & 0,0 & $-0,45$ & 1,24 & $-0,01$ \\
\hline Écart & $-1,3^{\star \star}$ & $11,0^{\star \star}$ & $-2,0^{\star *}$ & $-0,06^{* *}$ & $0,44^{\star *}$ & $-0,06^{\star \star}$ \\
\hline Student & 2,15 & 11,41 & 3,99 & 2,25 & 12,63 & 3,83 \\
\hline \multicolumn{7}{|l|}{$t+2$} \\
\hline Femmes & $-7,6$ & 27,5 & $-0,3$ & $-0,40$ & 0,92 & $-0,02$ \\
\hline Hommes & $-8,7$ & 23,4 & 1,1 & $-0,45$ & 0,77 & 0,05 \\
\hline Écart & 1,1 & $4,2^{\star \star}$ & $-1,3^{\star \star}$ & $0,05^{\star}$ & $0,15^{\star \star}$ & $-0,07^{\star \star}$ \\
\hline Student & 1,56 & 4,39 & 2,04 & 1,77 & 4,43 & 2,77 \\
\hline \multicolumn{7}{|l|}{$t+3$} \\
\hline Femmes & $-9,0$ & 16,3 & 0,9 & $-0,42$ & 0,45 & 0,03 \\
\hline Hommes & $-9,8$ & 15,7 & 2,5 & $-0,50$ & 0,44 & 0,08 \\
\hline Écart & 0,8 & 0,7 & $-1,6^{\star *}$ & $0,07^{\star \star}$ & 0,01 & $-0,05^{\star}$ \\
\hline Student & 0,94 & 0,71 & 2,07 & 2,10 & 0,42 & 1,79 \\
\hline \multicolumn{7}{|l|}{$t+4$} \\
\hline Femmes & $-11,0$ & 6,3 & 1,2 & $-0,47$ & 0,17 & 0,05 \\
\hline Hommes & $-13,2$ & 8,0 & 2,7 & $-0,59$ & 0,20 & 0,09 \\
\hline Écart & $2,2^{\star \star}$ & $-1,7^{\star \star}$ & $-1,5^{\star}$ & $0,12^{\star \star}$ & $-0,03$ & $-0,04$ \\
\hline Student & 2,21 & 2,07 & 1,81 & 2,88 & 1,40 & 1,28 \\
\hline \multicolumn{7}{|l|}{$t+5$} \\
\hline Femmes & $-12,8$ & 5,6 & 0,4 & $-0,54$ & 0,17 & 0,04 \\
\hline Hommes & $-12,6$ & 8,3 & 2,6 & $-0,55$ & 0,21 & 0,09 \\
\hline Écart & $-0,2$ & $-2,7^{\star \star}$ & $-2,2^{\star \star}$ & 0,00 & $-0,04$ & $-0,05$ \\
\hline Student & 0,20 & 3,06 & 2,43 & 0,03 & 1,60 & 1,63 \\
\hline
\end{tabular}

Note : comparaison de l'effet du cancer sur la situation professionnelle selon le genre. Les effets sont repris du tableau $5 \mathrm{~A}^{* \star}:$ significatif au seuil de $5 \%$; ${ }^{*}$ : significatif au seuil de $10 \%$.

Lecture : un an après l'apparition du cancer, les hommes sont 49,9 \% à cotiser en maladie et les femmes sont 38,9\%. L'écart entre ces deux pourcentages est de $11 \%$, il est statistiquement significatif au seuil de $5 \%$.

Champ : tout l'échantillon.

Source : Hygie (Irdes). 
Tableau 6

Effet du cancer sur l'activité professionnelle, en fonction de l'âge auquel il survient - Femmes

\begin{tabular}{|c|c|c|c|c|c|c|c|}
\hline & \multirow{2}{*}{$\begin{array}{c}\text { Nombre de traités } \\
\text { Taux d'appariement (en \%) }\end{array}$} & \multicolumn{3}{|c|}{ Situation (en \%) } & \multicolumn{3}{|c|}{ Durée (en trimestres) } \\
\hline & & Emploi & Maladie & Chômage & Emploi & Maladie & Chômage \\
\hline \multicolumn{8}{|c|}{ Femmes $<48$ ans } \\
\hline \multicolumn{8}{|l|}{$t-1, t+1$} \\
\hline$E\left(y_{0} \mid T=1\right)$ & 2660 & 97,2 & 0,9 & 2,9 & 3,76 & 0,02 & 0,09 \\
\hline Effet & 99,8 & $-7,1^{\star \star}$ & $46,9^{\star \star}$ & $-1,7^{\star *}$ & $-0,45^{\star *}$ & $1,60^{\star \star}$ & $-0,06^{\star *}$ \\
\hline Student & & 14,06 & 49,88 & 4,09 & 18,95 & 49,62 & 4,24 \\
\hline \multicolumn{8}{|l|}{$t-1, t+2$} \\
\hline$E\left(y_{0} \mid T=1\right)$ & 2410 & 97,1 & 0,9 & 2,7 & 3,75 & 0,02 & 0,08 \\
\hline Effet & 99,8 & $-5,6^{\star \star}$ & $24,8^{\star \star}$ & 0,3 & $-0,29^{\star \star}$ & $0,85^{\star \star}$ & 0,00 \\
\hline Student & & 10,30 & 28,96 & 0,30 & 12,42 & 28,55 & 0,42 \\
\hline \multicolumn{8}{|l|}{$t-1, t+3$} \\
\hline$E\left(y_{0} \mid T=1\right)$ & 2210 & 97,0 & 0,9 & 2,7 & 3,75 & 0,02 & 0,08 \\
\hline Effet & 99,8 & $-5,6^{\star \star}$ & $14,9^{\star \star}$ & $1,6^{\star \star}$ & $-0,29^{\star *}$ & $0,44^{\star \star}$ & $0,05^{\star \star}$ \\
\hline Student & & 9,28 & 18,55 & 2,26 & 11,45 & 18,72 & 2,04 \\
\hline \multicolumn{8}{|l|}{$t-1, t+4$} \\
\hline$E\left(y_{0} \mid T=1\right)$ & 1968 & 96,7 & 0,8 & 2,7 & 3,74 & 0,02 & 0,08 \\
\hline Effet & 99,8 & $-7,3^{\star \star}$ & $7,7^{\star *}$ & $1,4^{*}$ & $-0,31^{\star *}$ & $0,22^{\star \star}$ & $0,05^{\star \star}$ \\
\hline Student & & 9,81 & 10,31 & 1,85 & 9,80 & 10,94 & 2,24 \\
\hline \multicolumn{8}{|l|}{$t-1, t+5$} \\
\hline$E\left(y_{0} \mid T=1\right)$ & 1774 & 96,5 & 0,8 & 2,6 & 3,72 & 0,02 & 0,08 \\
\hline Effet & 99,8 & $-8,0^{\star \star}$ & $6,8^{* *}$ & $-0,1$ & $-0,36^{\star *}$ & $0,21^{\star \star}$ & 0,01 \\
\hline Student & & 10,13 & 8,86 & 0,33 & 10,54 & 9,68 & 0,25 \\
\hline \multicolumn{8}{|c|}{ Femmes $>=48$ ans } \\
\hline \multicolumn{8}{|l|}{$t-1, t+1$} \\
\hline$E\left(y_{0} \mid T=1\right)$ & 2877 & 94,7 & 0,3 & 3,9 & 3,71 & 0,01 & 0,14 \\
\hline Effet & 98,3 & $-9,2^{\star \star}$ & $52,8^{\star *}$ & $-2,3^{\star \star}$ & $-0,56^{\star *}$ & $1,75^{\star \star}$ & $-0,09^{\star *}$ \\
\hline Student & & 15,24 & 60,85 & 5,39 & 21,86 & 54,41 & 5,54 \\
\hline \multicolumn{8}{|l|}{$t-1, t+2$} \\
\hline$E\left(y_{0} \mid T=1\right)$ & 2383 & 95,4 & 0,3 & 3,2 & 3,74 & 0,01 & 0,11 \\
\hline Effet & 98,2 & $-9,6^{\star \star}$ & $30,4^{\star \star}$ & $-0,9$ & $-0,51^{\star *}$ & $0,99^{\star \star}$ & $-0,03$ \\
\hline Student & & 13,44 & 31,85 & 1,57 & 17,96 & 31,55 & 1,46 \\
\hline \multicolumn{8}{|l|}{$t-1, t+3$} \\
\hline$E\left(y_{0} \mid T=1\right)$ & 1996 & 95,8 & 0,3 & 2,8 & 3,76 & 0,01 & 0,09 \\
\hline Effet & 98,5 & $-12,8^{\star \star}$ & $17,9^{\star \star}$ & 0,0 & $-0,58^{\star *}$ & $0,46^{\star \star}$ & 0,01 \\
\hline Student & & 14,60 & 19,98 & 0,03 & 16,34 & 17,74 & 0,22 \\
\hline \multicolumn{8}{|l|}{$t-1, t+4$} \\
\hline$E\left(y_{0} \mid T=1\right)$ & 1613 & 96,0 & 0,3 & 2,3 & 3,77 & 0,01 & 0,08 \\
\hline Effet & 98,6 & $-15,5^{\star \star}$ & $4,5^{\star \star}$ & 0,9 & $-0,67^{\star *}$ & $0,12^{\star \star}$ & 0,05 \\
\hline Student & & 14,38 & 6,52 & 0,97 & 15,65 & 6,38 & 1,47 \\
\hline \multicolumn{8}{|l|}{$t-1, t+5$} \\
\hline$E\left(y_{0} \mid T=1\right)$ & 1289 & 95,9 & 0,3 & 2,2 & 3,76 & 0,01 & 0,07 \\
\hline Effet & 98,5 & $-19,4^{\star \star}$ & $4,0^{\star *}$ & 1,1 & $-0,79^{\star *}$ & $0,13^{\star \star}$ & $0,07^{\star}$ \\
\hline Student & & 14,95 & 5,04 & 1,08 & 15,51 & 5,55 & 1,76 \\
\hline
\end{tabular}

Note : effet d'un cancer sur l'activité. Différence des différences avec appariement exact. Les $t$ de Student sont estimés par le bootstrap par grappe sur 500 répétitions. Variables d'appariement : indicatrices d'emploi, de maladie et de chômage en $t-1$, année de naissance, genre, quintile de salaire en début de carrière. ${ }^{\star \star}$ : significatif au seuil de $5 \% ;{ }^{*}$ : significatif au seuil de $10 \%$.

Lecture : pour chaque genre, le tableau mesure l'effet moyen du cancer sur les individus atteints entre une année avant la survenue de la maladie et $k$ années après celle-ci, $\mathrm{k}=1, \ldots 5$. Les trois premières lignes de la colonne Emploi renseignent respectivement sur le fait que $97,2 \%$ des femmes avec un cancer de moins de 48 ans étaient en emploi au moins un trimestre un an avant le cancer, que l'effet de la survenue du cancer est de - 7,1 points de pourcentage, significativement différent de 0 pour une statistique de Student de 14,06. Champ : femmes de l'échantillon.

Source : Hygie (Irdes). 
Tableau 7

Effet du cancer sur l'activité professionnelle, en fonction de l'âge auquel il survient - Hommes

\begin{tabular}{|c|c|c|c|c|c|c|c|}
\hline & \multirow{2}{*}{\begin{tabular}{|c|} 
Nombre de traités \\
Taux d'appariement (en \%)
\end{tabular}} & \multicolumn{3}{|c|}{ Situation (en \%) } & \multicolumn{3}{|c|}{ Durée (en trimestres) } \\
\hline & & Emploi & Maladie & Chômage & Emploi & Maladie & Chômage \\
\hline \multicolumn{8}{|c|}{ Hommes $<51$ ans } \\
\hline \multicolumn{8}{|l|}{$t-1, t+1$} \\
\hline$E\left(y_{0} \mid T=1\right)$ & 2121 & 99,1 & 0,2 & 1,6 & 3,91 & 0,00 & 0,05 \\
\hline Effet & 99,2 & $-7,5^{\star \star}$ & $38,4^{\star \star}$ & $-0,3$ & $-0,47^{\star *}$ & $1,23^{\star \star}$ & $-0,02$ \\
\hline Student & & 12,84 & 38,04 & 0,66 & 17,87 & 35,01 & 1,49 \\
\hline \multicolumn{8}{|l|}{$t-1, t+2$} \\
\hline$E\left(y_{0} \mid T=1\right)$ & 1904 & 99,0 & 0,2 & 1,5 & 3,90 & 0,00 & 0,04 \\
\hline Effet & 99,1 & $-8,7^{\star \star}$ & $22,0^{\star \star}$ & $1,3^{\star}$ & $-0,45^{\star \star}$ & $0,74^{\star \star}$ & $0,06^{\star \star}$ \\
\hline Student & & 12,73 & 23,28 & 1,95 & 15,50 & 22,49 & 2,49 \\
\hline \multicolumn{8}{|l|}{$t-1, t+3$} \\
\hline$E\left(y_{0} \mid T=1\right)$ & 1727 & 98,9 & 0,2 & 1,5 & 3,90 & 0,00 & 0,04 \\
\hline Effet & 99,0 & $-9,1^{* *}$ & $15,8^{\star \star}$ & $3,1^{\star *}$ & $-0,46^{* *}$ & $0,44^{\star \star}$ & $0,09^{\star \star}$ \\
\hline Student & & 11,99 & 18,20 & 4,76 & 14,68 & 17,04 & 4,30 \\
\hline \multicolumn{8}{|l|}{$t-1, t+4$} \\
\hline$E\left(y_{0} \mid T=1\right)$ & 1587 & 98,8 & 0,2 & 1,6 & 3,90 & 0,00 & 0,05 \\
\hline Effet & 99,0 & $-11,9^{\star \star}$ & $7,5^{\star *}$ & $3,1^{\star *}$ & $-0,53^{* *}$ & $0,18^{\star \star}$ & $0,10^{\star *}$ \\
\hline Student & & 13,14 & 10,01 & 4,16 & 14,57 & 9,60 & 4,06 \\
\hline \multicolumn{8}{|l|}{$t-1, t+5$} \\
\hline$E\left(y_{0} \mid T=1\right)$ & 1419 & 98,8 & 0,2 & 1,5 & 3,89 & 0,00 & 0,04 \\
\hline Effet & 98,9 & $-11,7^{\star \star}$ & $7,6^{\star *}$ & $2,4^{\star *}$ & $-0,51^{\star *}$ & $0,19^{\star \star}$ & $0,08^{\star \star}$ \\
\hline Student & & 12,12 & 9,61 & 3,25 & 12,95 & 8,62 & 2,99 \\
\hline \multicolumn{8}{|c|}{ Hommes $>=51$ ans } \\
\hline \multicolumn{8}{|l|}{$t-1, t+1$} \\
\hline$E\left(y_{0} \mid T=1\right)$ & 2504 & 94,9 & 0,3 & 4,5 & 3,77 & 0,01 & 0,17 \\
\hline Effet & 97,7 & $-6,1^{\star \star}$ & $39,5^{\star *}$ & 0,2 & $-0,41^{\star \star}$ & $1,25^{\star \star}$ & 0,01 \\
\hline Student & & 9,17 & 37,84 & 0,33 & 13,90 & 34,94 & 0,35 \\
\hline \multicolumn{8}{|l|}{$t-1, t+2$} \\
\hline$E\left(y_{0} \mid T=1\right)$ & 1880 & 96,1 & 0,4 & 3,1 & 3,82 & 0,01 & 0,11 \\
\hline Effet & 97,7 & $-8,7^{\star \star}$ & $25,3^{\star \star}$ & 0,7 & $-0,46^{\star \star}$ & $0,82^{\star \star}$ & 0,04 \\
\hline Student & & 10,05 & 22,59 & 0,86 & 12,73 & 21,18 & 1,34 \\
\hline \multicolumn{8}{|l|}{$t-1, t+3$} \\
\hline$E\left(y_{0} \mid T=1\right)$ & 1430 & 96,8 & 0,3 & 2,2 & 3,85 & 0,01 & 0,08 \\
\hline Effet & 97,6 & $-11,1^{\star \star}$ & $15,4^{\star \star}$ & 1,4 & $-0,56^{\star \star}$ & $0,44^{\star \star}$ & 0,06 \\
\hline Student & & 9,67 & 13,66 & 1,41 & 12,24 & 12,31 & 1,48 \\
\hline \multicolumn{8}{|l|}{$t-1, t+4$} \\
\hline$E\left(y_{0} \mid T=1\right)$ & 1038 & 97,3 & 0,3 & 1,6 & 3,87 & 0,01 & 0,05 \\
\hline Effet & 97,4 & $-15,7^{\star \star}$ & $8,9^{\star \star}$ & 2,0 & $-0,70^{\star *}$ & $0,24^{\star \star}$ & 0,07 \\
\hline Student & & 10,58 & 7,77 & 1,36 & 11,71 & 7,55 & 1,26 \\
\hline \multicolumn{8}{|l|}{$t-1, t+5$} \\
\hline$E\left(y_{0} \mid T=1\right)$ & 772 & 97,6 & 0,3 & 1,3 & 3,88 & 0,01 & 0,04 \\
\hline Effet & 97,4 & $-14,9^{\star \star}$ & $9,9^{\star *}$ & $3,1^{*}$ & $-0,64^{\star \star}$ & $0,28^{\star \star}$ & $0,12^{\star *}$ \\
\hline Student & & 9,05 & 7,24 & 1,95 & 9,70 & 6,87 & 2,00 \\
\hline
\end{tabular}

Note : effet d'un cancer sur l'activité. Différence des différences avec appariement exact. Les $t$ de Student sont estimés par le bootstrap par grappe sur 500 répétitions. Variables d'appariement : indicatrices d'emploi, de maladie et de chômage en $t-1$, année de naissance, genre, quintile de salaire en début de carrière. ${ }^{\star \star}$ : significatif au seuil de $5 \% ;{ }^{*}$ : significatif au seuil de $10 \%$.

Lecture : pour chaque genre, le tableau mesure l'effet moyen du cancer sur les individus atteints entre une année avant la survenue de la maladie et $k$ années après celle-ci, $\mathrm{k}=1, \ldots .5$. Les trois premières lignes de la colonne Emploi renseignent respectivement sur le fait que $99,1 \%$ des hommes avec un cancer traités de moins de 51 ans étaient en emploi au moins un trimestre un an avant le cancer, que l'effet de la survenue du cancer est de - 7,5 points de pourcentage, significativement différent de 0 pour une statistique de Student de 12,84. Champ : hommes de l'échantillon.

Source : Hygie (Irdes). 
Graphique I

Effet du cancer sur la présence en emploi et en maladie, selon le quintile de salaires à l'entrée sur le marché du travail
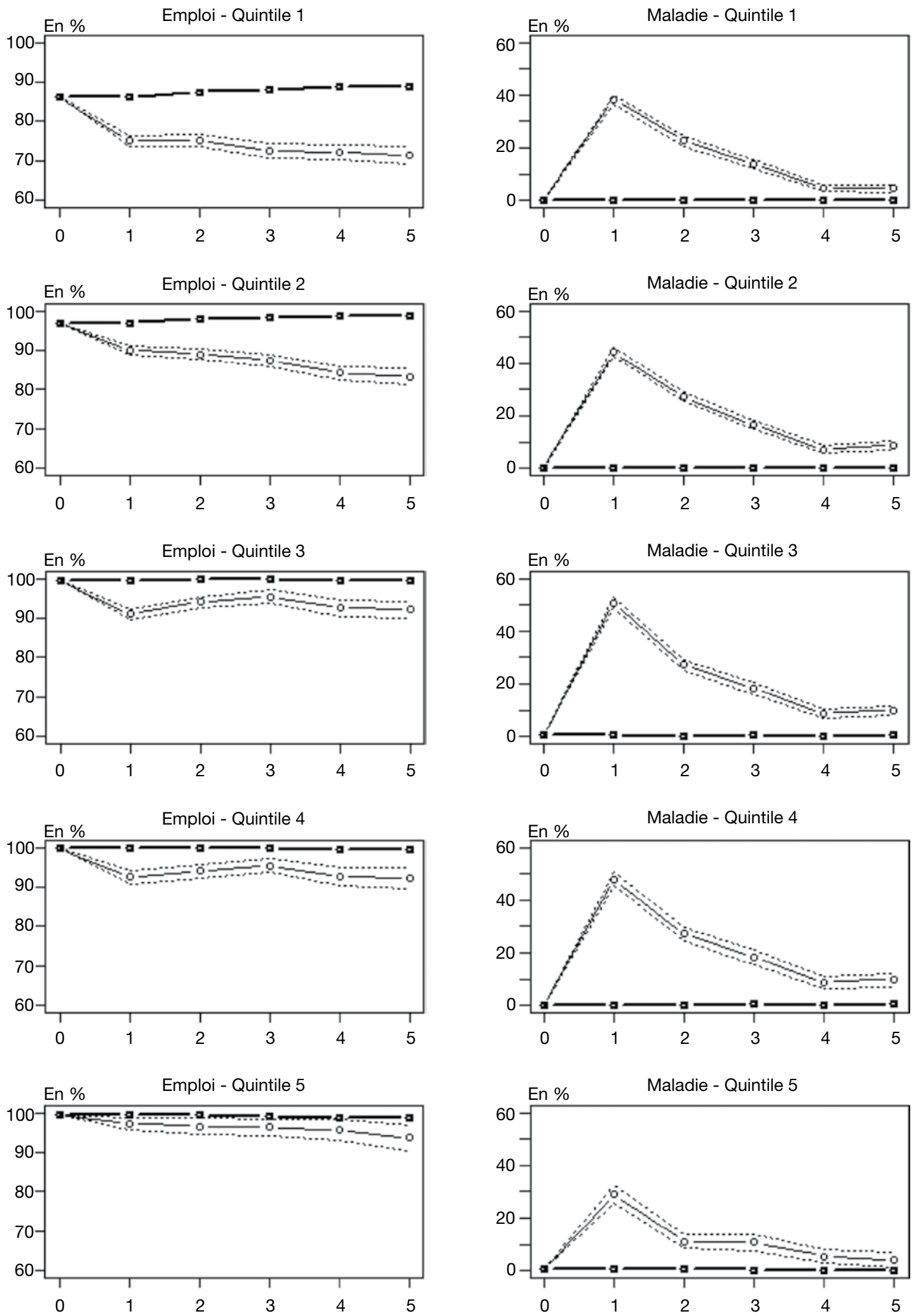

Note : le trait gras indique la valeur de la variable pour les non-traités, le trait plein sa valeur pour les traités de $t+1$ à $t+5$. Les traits en pointillés indiquent l'intervalle de confiance de niveau $95 \%$ pour les traités.

Lecture : à peu près $90 \%$ des personnes sont en emploi dans le premier quintile de revenu initial, un an avant l'apparition d'un cancer. Un an après le taux d'emploi est de $75 \%$, cinq ans après il est de $70 \%$, toutes choses égales par ailleurs. Dans le dernier quintile de revenu, le taux d'emploi est proche de $100 \%$ en l'absence de cancer, il est de $95 \%$ cinq ans après l'apparition du cancer.

Champ : tout l'échantillon.

Source : Hygie (Irdes) 
Graphique II

\section{Effet du cancer sur le nombre de trimestres validés en emploi et en maladie, selon le quintile} de salaires à l'entrée sur le marché du travail
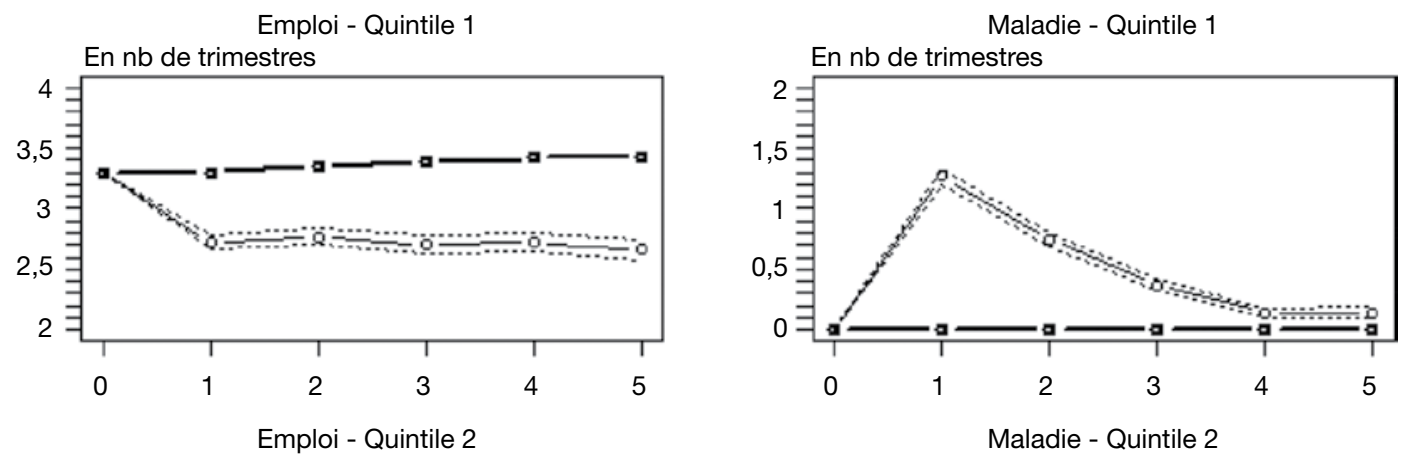

En nb de trimestres
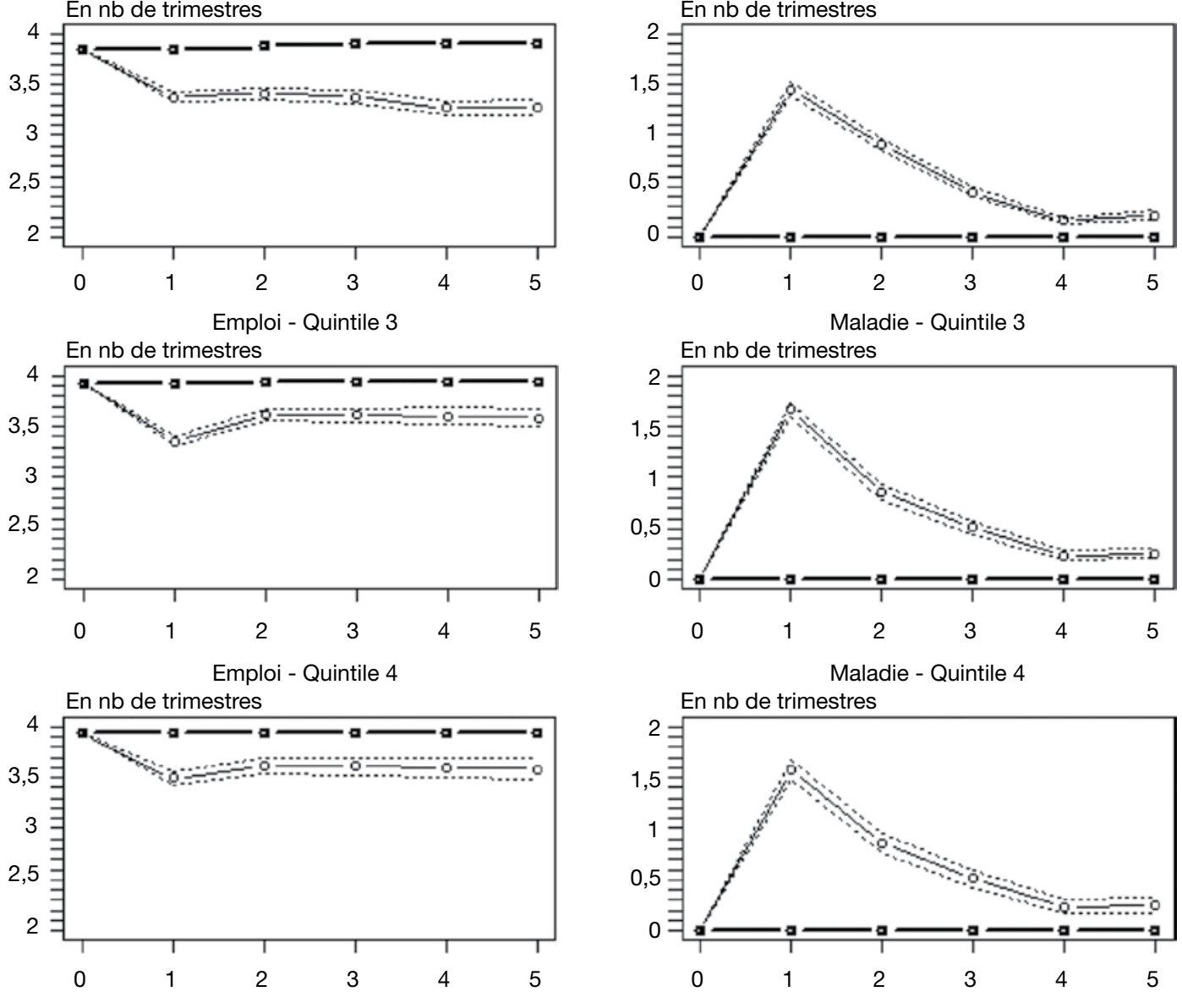

Emploi - Quintile 5
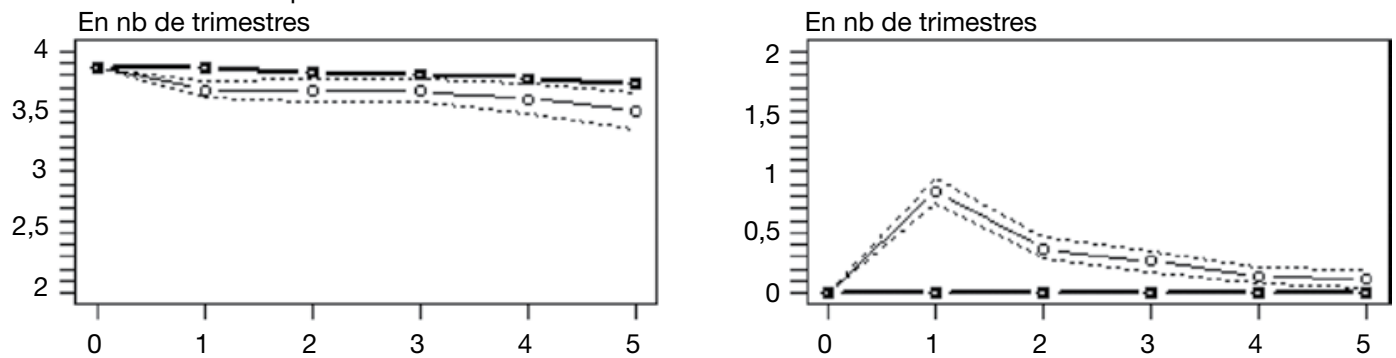

Note : le trait gras indique la valeur de la variable pour les non-traités, le trait plein sa valeur pour les traités de $t+1$ à $t+5$. Les traits en pointillés indiquent l'intervalle de confiance de niveau $95 \%$ pour les traités.

Lecture : les personnes du premier quintile de revenu initial ne valident quasiment pas de trimestre en maladie un an avant l'apparition du cancer. Après un an, ils valident 1,5 trimestres, et ce chiffre retourne à 0 à partir de la quatrième année. Dans le dernier quintile de revenu initial, les travailleurs valident un seul trimestre en maladie et ce chiffre s'annule également à partir de la quatrième année qui suit le cancer.

Champ : tout l'échantillon

Source : Hygie (Irdes). 
« jeunes » (moins de 51 ans) et les hommes « âgés » (51 ans et plus).

Parmi les « jeunes », on observe également un effet négatif du cancer sur la situation d'emploi : à un an, la proportion d'hommes en situation d'emploi diminue de 7,5 pp passant de $99,1 \%$ à $91,6 \%$; celle des femmes diminue de 7,1 points passant de $97,2 \%$ à $90,1 \%$. L'avancée en âge ne semble cependant pas renforcer significativement l'effet négatif de la survenue du cancer sur l'emploi de court terme (à un an). Ces effets sont en effet de 9,2 pp pour les femmes «âgées » et de 6,1 pp pour les hommes « âgés ». Ce faible écart entre les plus jeunes et les plus âgés s'explique par le caractère radical et incompressible des traitements du cancer quel que soit l'âge : les traitements imposent de façon inconditionnelle, dans un premier temps, une mise à distance de l'emploi.

Parmi les personnes plus âgées, l'effet du cancer à court terme sur la situation professionnelle semble moins fort pour les hommes que pour les femmes. Cet écart pourrait résulter de situations sur le marché du travail moins sécurisées pour les femmes.

Par ailleurs, les différences selon le niveau de salaire à l'entrée du marché du travail semblent nettement défavoriser le premier quintile par rapport aux autres et surtout par rapport au dernier quintile (voir graphique I). Ainsi, une année après la survenue du cancer, on constate une diminution de la probabilité d'être en emploi de 11 pp versus une diminution de 6,9 à 8,4 pp pour les trois quintiles suivants, et surtout nettement plus faible pour le dernier quintile (2,2 pp).

\section{L'emploi à moyen terme}

En examinant l'effet du cancer sur une temporalité plus longue, nous constatons une amplification du phénomène de rupture sur le marché du travail : en $t+5$ l'effet est respectivement de 12,6 et 12,8 pp pour les hommes et les femmes. Par rapport à la situation de court terme, plus détériorée pour les malades femmes, la situation d'emploi à moyen terme est plus désavantageuse pour les hommes, mais leur probabilité d'emploi n'est significativement plus faible qu'en $t+4$ (voir tableau 5B).

De plus, l'âge de survenue du cancer devient une variable clé pouvant expliquer les différences de réinsertion professionnelle. En effet, la situation de moyen terme vis-à-vis de l'emploi est nettement plus détériorée pour les plus âgés : en $t+5$, la proportion d'hommes « âgés »

Encadré 4

\section{GRILLE DE LECTURE DES RÉSULTATS DES RÉGRESSIONS}

Les tableaux $5 \mathrm{~A}$ et $5 \mathrm{~B}$ permettent de distinguer les effets de court (un an) et moyen termes (de deux à cinq ans). Les résultats prennent en compte le rôle de la situation professionnelle passée sur la trajectoire professionnelle après la survenue du cancer via l'introduction des variables endogènes retardées. La lecture aisée de ces tableaux nécessite certains rappels concernant les principes de la méthode d'estimation utilisée.

Le tableau $5 \mathrm{~A}$ présente les estimations avec appariement exact selon le genre. Le tableau $5 \mathrm{~B}$ permet d'apprécier la significativité des écarts constatés entre les hommes et les femmes. Les résultats reportés dans ces deux tableaux sont obtenus au moyen de la méthode d'estimation de base qui autorise des effets individuels et temporels corrélés de sorte qu'elle est robuste aux variables omises qui ne varient que dans ces deux dimensions prises séparément, et aux produits croisés entre les variables d'appariement et les effets temporels. Toutefois, si les variables omises variaient de façon concomitante dans les deux dimensions, temporelles et individuelles, nos estimations pourraient ne pas être convergentes. Nous avons donc ajouté les variables endogènes retardées parmi les variables d'appariement pour améliorer la robustesse de nos estimations.

Un autre résultat, valable pour l'ensemble des estimations est la valeur souvent élevée des $t$ de Student asymptotiques : souvent, même la troisième décimale des effets estimés est significative. Cette propriété provient du nombre d'observations utilisées. Une estimation peut reposer sur plusieurs millions d'observations et la variance asymptotique d'un estimateur décroît avec le nombre d'observations de l'échantillon (plus précisément, la variance de $\sqrt{N}(\hat{\gamma}-\gamma)$ tend vers une matrice finie quand $N$ tend vers l'infini). II en résulte que le $t$ de Student décroît avec la racine carrée du nombre d'observations. Concrètement, quasiment toutes nos estimations sont significatives aux seuils usuels, ce qui nous autorise à interpréter nos coefficients directement. Ceci nous amène aussi à considérer directement l'importance quantitative des coefficients. Deux coefficients très proches peuvent être statistiquement différents et malgré tout présenter des valeurs économiquement équivalentes (e.g., $-0,14$ et $-0,15)$. Dans ce cas, nous les commentons comme s'ils étaient égaux. 
en emploi chute de 14,9 pp tandis qu'elle diminue de 19,4 pp pour les femmes «âgées » contre une diminution de $11,7 \mathrm{pp}$ pour les hommes « jeunes » et de 8 pp pour les femmes « jeunes » (cf. tableaux 6 et 7$)$.

Cette baisse de la probabilité d'exercer un emploi à moyen terme pour les individus plus âgés peut s'expliquer par un phénomène de double peine qui per se peut conduire à des effets amplifiés d'exclusion du marché du travail. Les analyses traditionnelles relatives aux investissements en capital humain et de santé peuvent être renforcées par des phénomènes de modifications de préférences ou de discrimination liées à l'âge - que l'on peut difficilement contrôler avec nos données mais qui ont été traditionnellement soulevés, en particulier pour les travailleurs les plus âgés, dans la littérature économique sur les effets d'une maladie chronique sur le marché du travail (voir par exemple Datta Gupta et Larsen, 2010).

Quoi qu'il en soit, il est attesté que les plus de 50 ans se caractérisent en moyenne par une probabilité de conserver ou de retourner à l'emploi plus faible que les plus jeunes.

Ensuite, les conséquences des traitements (dont certaines peuvent apparaître avec retard) et la co-morbidité associée peuvent renforcer les séquelles et accentuer, plus globalement, la dépréciation du capital de santé avec des phénomènes plus fréquents d'irréversibilité. Pour les femmes, les traitements du cancer du sein, lorsqu'il est hormono-dépendant par exemple, sont des traitements d'hormonothérapie de cinq ans la plupart du temps et susceptibles d'entrainer des douleurs articulaires importantes venant s'ajouter aux séquelles dites du «gros bras» fréquents après la chirurgie axillaire du cancer du sein. Les femmes plus âgées semblent victimes d'un effet multiplicateur; le cumul de facteurs de fragilité face à l'emploi conduit à un éloignement durable du marché du travail et croissant avec le temps $(9,2 \mathrm{pp}$ en $t+1,9,6 \mathrm{pp}$ en $t+2,12,8 \mathrm{pp}$ en $t+3$, 15,5 pp en $t+4$ et 19,4 pp en $t+5$, cf. tableau 6 ).

Si l'avancée en âge et le fait d'être une femme pénalisent les capacités de maintien sur le marché du travail à moyen terme des survivants du cancer, le salaire à l'entrée sur le marché du travail joue aussi un rôle déterminant qui vient amplifier le choc de court terme. Le décrochage des personnes dont les niveaux de salaires à l'entrée sur le marché du travail sont les plus faibles (quintiles 1 et 2) s'accroît dans le temps, alors qu'il est contenu à des niveaux sensiblement plus faibles pour les tranches de revenus les plus élevées. Cinq ans après la survenue du cancer, la sortie d'emploi est à peu près trois fois plus fréquente dans les deux premiers quintiles (respectivement - 17,4 pp et - 15,4 pp) que dans le dernier $(-4,9 \mathrm{pp})$.

\section{Les arrêts maladie et le chômage}

Les effets du cancer sur les arrêts maladie sont très élevés la première année suivant l'enregistrement administratif du cancer en ALD. L'accroissement du pourcentage d'individus avec au moins un trimestre en congé maladie est très important $(+49,9 \mathrm{pp}$ chez les femmes et $+38,9$ pp chez les hommes) en raison des traitements médicaux reçus, ceux-ci étant souvent longs lorsque se cumulent par exemple chirurgie, radiothérapie et chimiothérapie ( $c f$. tableau 5A). L'effet moyen sur l'occurrence d'être en arrêt de travail pour maladie diminue régulièrement pendant les trois premières années pour atteindre un minimum après quatre années. Par rapport à la situation initiale une année avant la déclaration en ALD cancer, la probabilité d'être en arrêt maladie est, pour les femmes atteintes d'un cancer, supérieure de 27,5 pp la deuxième année, de $16,3 \mathrm{pp}$ la troisième année et seulement de 6,3 et $5,6 \mathrm{pp}$ les quatrième et cinquième années. Chez les hommes, cette diminution est aussi marquée passant d'un effet moyen du cancer la deuxième année égal à $23,4 \mathrm{pp}$, la troisième année à $15,7 \mathrm{pp}$ pour atteindre un plancher d'environ $8 \mathrm{pp}$ les quatrième et cinquième années. Le tableau $5 \mathrm{~B}$ montre que cette diminution de la probabilité d'arrêt maladie est plus forte pour les femmes sur cet horizon de cinq ans. En effet, alors que les malades femmes sont significativement plus souvent arrêtées que les malades hommes un et deux ans après la survenue du cancer, cette situation s'inverse ensuite puisque ce sont les malades hommes qui sont significativement plus souvent en arrêt maladie après quatre et cinq ans.

L'accroissement du pourcentage d'individus en arrêt maladie est élevé la première année pour les jeunes (46,9 pp chez les femmes et 38,4 pp chez les hommes) comme pour les plus âgés $(52,8 \mathrm{pp}$ chez les femmes et $39,5 \mathrm{pp}$ chez les hommes). À nouveau, les femmes plus âgées semblent davantage en proie à l'effet pénalisant du cancer avec un taux d'arrêts maladie de $6 \mathrm{pp}$ supérieur à celui des plus jeunes contre 1,1 pp pour les hommes (cf. tableaux 6 et 7). Au fil du temps, le cancer continue à avoir un effet favorisant la situation d'arrêt maladie mais qui décroît dans le temps. Ainsi 
après cinq années, la probabilité de transition vers la maladie a sensiblement diminué. Elle correspond, chez les jeunes, à une augmentation de 7,6 pp pour les hommes et 6,8 pp pour les femmes et, chez les plus âgés, de 9,9 pp pour les hommes et $4 \mathrm{pp}$ chez les femmes.

Dans le court terme, les durées d'arrêt maladie sont plus courtes pour les individus entrés sur le marché du travail avec un salaire appartenant au premier et surtout au dernier quintile de la distribution des salaires. L'analyse de moyen terme de ces résultats en termes d'arrêt maladie confirme les différences entre quintiles observés. Si les effets du cancer s'estompent pour toutes les catégories de niveaux de salaires à l'entrée sur le marché du travail, les quintiles 2 à 4 restent caractérisés par les accroissements les plus forts de la probabilité d'arrêt maladie (entre 8,5 et $9,3 \mathrm{pp}$ ) alors que cet effet pour les classes de salaires extrêmes est moitié moins élevé environ.

La déclaration du cancer en ALD cancer implique également une diminution de la situation de chômage l'année qui suit la survenue du cancer chez les femmes (de 2 pp passant de $3,4 \%$ à $1,4 \%$ ). Deux explications peuvent être avancées. D'une part, certains chômeurs ont pu transiter vers une situation d'inactivité, étant rendus inaptes à la recherche active d'emploi, et, d'autre part, on peut faire l'hypothèse d'un effet de substitution entre situations professionnelles (transition de la situation de chômage indemnisé vers l'arrêt maladie) en partie du fait des règles administratives. Ainsi, afin de valider un trimestre en chômage, il convient d'être demandeur d'emploi, c'est-à-dire en situation de recherche effective et permanente d'un emploi. Dès lors que l'individu est déclaré en arrêt maladie, il ne peut plus être simultanément enregistré comme chômeur. Bien sûr, les indemnités maladie viennent se substituer aux indemnités chômage et les droits du chômeur reprendront effet à la fin de la période d'arrêt maladie. Mais en termes de trimestres validés, il est normal d'observer un mécanisme partiel de substitution entre « chômage » et « maladie » pour des maladies impliquant des traitements lourds et des arrêts longs. Au-delà d'une année, l'effet du cancer sur le chômage est quasiment nul chez les femmes et croît très légèrement pour les hommes (hausse de 2,4 pp de la probabilité d'être au chômage quatre et cinq ans après). Cet effet est peu différencié par âge : au fil du temps, le pourcentage d'individus en situation de chômage augmente peu, les individus étant vraisemblablement en inactivité et, pour certains, et notamment les hommes en situation d'arrêt maladie.

\section{La durée de la situation professionnelle post cancer selon le genre}

Le ratio de durée des situations post cancer permet d'approfondir les résultats précédents en estimant le nombre de jours passés, notamment en arrêts maladie (selon l'âge d'inscription en ALD et le salaire à l'entrée sur le marché du travail) et au chômage.

\section{La durée d'arrêt maladie}

La durée de l'arrêt maladie, un an après la survenue du cancer, augmente de 1,68 trimestre pour les femmes et de 1,24 trimestre pour les hommes. La différence observée entre hommes et femmes peut s'expliquer par le caractère plus invalidant de certains traitements des cancers féminins ${ }^{14}$. À moyen terme, l'effet du cancer sur la durée d'arrêt maladie s'estompe. Ainsi, pour les femmes, l'effet du cancer décroît de manière continue pendant les quatre premières années après le cancer pour se stabiliser à 0,17 trimestre d'absences supplémentaires. Pour les hommes, l'effet du cancer à $t+5$ est de 0,21 trimestre.

\section{... selon l'âge d'inscription en ALD}

Dans la population féminine, la survenue d'un cancer augmente la durée de l'arrêt maladie de 1,60 trimestre pour les plus jeunes à 1,75 trimestre pour les plus âgées (tableau 6). Cette augmentation de la durée en arrêt maladie avec l'âge de survenue du cancer n'est en revanche pas observée dans la population masculine $(1,23$ et 1,25 trimestre de plus en arrêt maladie respectivement pour les hommes jeunes et âgés) (cf. tableau 7). L'effet du cancer sur la durée d'arrêt maladie est plus persistant chez les personnes dont l'inscription en ALD intervient plus tardivement. Pour les jeunes comme pour les plus âgés, l'effet positif du cancer sur la durée moyenne d'arrêt maladie s'estompe avec le temps, mais on observe cependant des phénomènes distincts selon l'âge de survenue du cancer. Les femmes plus âgées vivent des périodes d'arrêt maladie plus longues que les jeunes femmes à court terme, en $t+1$ et l'année suivante en $t+2$ (l'écart en $t+2$ est ainsi de 0,14 trimestre,

14. Dans notre base, pour les femmes jeunes comme pour les femmes plus âgées, c'est le cancer du sein qui est le plus prévalent $(57,6 \%$ pour les femmes de plus de 48 ans et $41,8 \%$ pour les femmes de moins de 48 ans). Pour les hommes, le cancer le plus prévalent pour les plus âgés est le cancer de la prostate $(20,4 \%)$, suivi des tumeurs des bronches et du poumon $(11,9 \%)$ tandis que pour les hommes jeunes, c'est le cancer des testicules qui est le plus prévalent (18,5\%), suivi des tumeurs des bronches et du poumon (6,9 \%). 
cf. tableau 6). Cependant les courbes s'inversent au-delà de cet horizon semblant indiquer que les jeunes femmes ont un nombre de jours indemnisés pour maladie plus élevé que les femmes plus âgées. À moyen terme, en $t+5$, en effet, on observe un écart de trimestres validés en arrêt maladie de 0,08 trimestre pour les femmes jeunes. Il est possible qu'elles doivent faire face à des contraintes familiales plus fortes expliquant des arrêts maladie plus fréquents. Chez les hommes plus âgés, on note une nouvelle augmentation de la durée maladie en $t+5$ par rapport à l'année précédant le diagnostic du cancer (l'effet passe de 0,24 à 0,28 ). Si on compare la situation des hommes et des femmes d'âge supérieur à l'âge médian, celle des premiers apparaît plus affectée que celle des secondes : ceci peut traduire une sévérité et/ou des séquelles plus importantes du cancer touchant les hommes plus âgés comparativement à celui dont souffrent les femmes du même âge.

\section{... selon le salaire à l'entrée sur le marché du travail}

La stratification selon le niveau de salaires à l'entrée sur le marché du travail suggère une plus forte protection de la classe la plus aisée. En effet, l'effet du cancer pour les salariés appartenant au cinquième quintile se traduit par une augmentation de 0,87 du nombre de trimestres d'arrêt maladie alors que les effets dans les autres quintiles enregistrent une hausse des arrêts maladie de 1,26 à 1,65 trimestre (graphique II). Concernant le gradient de niveau de salaire à l'entrée sur le marché du travail, nous constatons une baisse année après année du nombre de trimestres en arrêts maladie pour les cinq quintiles. En revanche, le niveau et la vitesse de décroissance de la durée des arrêts maladie sont plus faibles pour le cinquième quintile, confirmant que les salariés les mieux rémunérés à leur entrée sur le marché du travail sont moins désavantagés face à la maladie, tant en termes de retour en emploi que de durée des interruptions pour cause de maladie. De tels effets de carrière différenciés selon le quintile de salaire à l'entrée sur le marché du travail peuvent également s'interpréter à l'aune de l'analyse de Grossman $(1972,2000)$ concernant l'effet de l'éducation sur la productivité des investissements en santé. En effet dans la modélisation de Grossman, l'investissement en santé aura un meilleur rendement pour les individus les plus éduqués; ce rendement peut être reflété par les résultats obtenus ici et qui expriment le plus faible désavantage face au cancer pour les individus ayant un salaire d'entrée sur le marché du travail plus élevé, corrélé en général au niveau d'éducation.

\section{La durée de chômage}

Le nombre de trimestres en chômage permet de nouveau d'évaluer la baisse du nombre de jours de chômage à la suite de la déclaration du cancer ( $c f$. tableau 5). L'année suivante, les femmes enregistrent une diminution de 0,07 trimestre passé en chômage; la baisse de 0,01 trimestre pour les hommes est en revanche négligeable. À moyen terme, on observe une augmentation de la durée de jours de chômage à partir de la deuxième année chez les hommes et de la quatrième année chez les femmes. L'effet de l'âge au diagnostic du cancer sur le nombre de trimestres en chômage varie quelque peu selon le genre (cf. tableaux 6 et 7). Ainsi, le nombre de trimestres en chômage diminue pour les jeunes femmes la première année puis augmente en $t+3$ et $t+4$. Pour les femmes âgées, le nombre de trimestres passés en chômage change de manière significative à deux horizons: il diminue en $t+1$ et augmente en $t+5$. Pour les jeunes hommes, le nombre de trimestres en chômage augmente à partir de $t+2$ de manière significative $(0,06$ trimestre) et régulièrement jusqu'en $t+4$. En revanche, pour les hommes plus âgés, la hausse du nombre de jours de chômage n'est significative qu'en $t+5$ : ils enregistrent 0,12 trimestre de chômage supplémentaire.

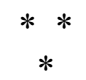

Nous avons considéré, dans cette étude la survenue du cancer, comme choc de santé exogèn $\mathrm{e}^{15}$. L'originalité de notre étude se situe à plusieurs niveaux. Tout d'abord, l'estimation de l'effet du cancer sur les situations professionnelles porte sur un horizon de un à cinq ans après l'enregistrement de l'individu dans le système administratif des ALD, contrairement aux autres études françaises. En outre, elle s'appuie sur des données administratives permettant de retracer les carrières d'un large échantillon de salariés du secteur privé, de

15. Nous ne pouvons pas totalement exclure l'endogénéité de la survenue du cancer du fait de préférences différentes pour le recours aux tests de dépistage liées au statut socio-économique. Toutefois, étant donné la prévalence des cancers dans notre base, le rôle des préférences pour le dépistage est probablement relativement mineur. Les dépistages systématiques, quelle que soit la catégorie socio-professionnelle d'appartenance des individus, sont peu réalisés avant 50 ans notamment pour le cancer du côlon. Dans notre base, constituée d'individus jeunes, on peut considérer que la question de l'endogénéité est minorée de ce fait. 
plus de 500000 bénéficiaires échantillonnés en 2005. Cette base de données permet de suivre ainsi les trajectoires professionnelles de plus de 14000 personnes atteintes d'un cancer. Ensuite, la taille de l'échantillon autorise une estimation par la méthode de différence des différences avec appariement exact. Ce dernier permet de disposer d'une population témoin jumelle de la population traitée en contrôlant rigoureusement de l'hétérogénéité observée. Enfin, nous examinons une population relativement jeune (de moins de 70 ans) en identifiant par conséquent des cancers survenus précocement, ce qui renforce la pertinence de l'analyse des trajectoires professionnelles de court et moyen termes pour cette population.

Nos principaux résultats confirment l'effet pénalisant du cancer sur l'emploi (diminution de la proportion d'individus ayant validé au moins un trimestre en emploi de respectivement 8 et $7 \mathrm{pp}$ pour les femmes et les hommes l'année suivante). La profondeur temporelle nous permet de juger de la persistance des effets de la maladie sur l'éloignement du marché du travail, voire de l'amplification du phénomène : à un horizon de cinq ans, la probabilité d'être employé au moins un trimestre dans l'année est ainsi plus faible de $13 \mathrm{pp}$ pour les personnes malades.

$\mathrm{Au}$ niveau français, ces résultats peuvent être mis en regard des résultats de l'enquête Drees-Inserm (2004) réalisée deux ans après le diagnostic. Cette base de données riche compile des données médicales, administratives et auto-déclarées. Elle comprend notamment des informations sur les conditions de vie (emploi, qualité de vie, revenus) et sur l'état de santé pour des individus ayant eu un diagnostic de cancer en 2002. Les études d'Eichenbaum-Voline et al. (2008) et Joutard et al. (2012) réalisent aussi un appariement entre l'échantillon des individus atteints par le cancer et la population générale des salariés décrite à partir de l'enquête Emploi de l'Insee. Après deux ans, ces deux études montrent une diminution de la probabilité d'être en emploi de 13 et $14 \mathrm{pp}$. Ces résultats illustrent donc une situation des malades face à l'emploi moins favorable que celle que nous estimons (respectivement une baisse de 8 et $9 \mathrm{pp}$ pour les femmes et les hommes). Cette différence mérite une discussion alors même que l'inclusion des indépendants dans ces deux études tend déjà à minorer la diminution du retour à l'emploi après le diagnostic (les indépendants étant moins bien couverts ont tendance à se maintenir le plus en emploi après un événement de santé).
Par ailleurs, Malavolti et al. (2008) étudient la reprise du travail selon le genre et constatent un retour à l'emploi plus rapide chez les hommes que chez les femmes entre le moment du diagnostic et la date de l'enquête. Les taux de retour à l'emploi après deux ans sont quasiment similaires pour les femmes et les hommes : $78 \%$ et $74 \%$ respectivement. Contrairement à ces études, nous ne pouvons pas analyser la vitesse de reprise d'emploi pour des intervalles infra-annuels, mais nos résultats montrent qu'après deux années l'effet sur la situation d'emploi est sensiblement similaire entre hommes et femmes.

Au total, il existe plusieurs sources d'explications des différences entre les études issues de l'enquête Drees-Inserm 2004 et la nôtre.

La première réside dans les indicateurs utilisés concernant la situation sur le marché du travail. Dans ces études, une mesure instantanée est réalisée à la date précise de l'entretien alors que nous mesurons la présence en emploi durant une année pour une durée au moins égale à trois mois.

La deuxième relève de la différence d'horizon temporel. Dans le cadre de notre échantillon, l'ancienneté du cancer peut, pour certains individus, être élevée et récente pour d'autres, ce qui signifie que nos évaluations de l'effet de la maladie sur la situation professionnelle concerne des dates différentes contrairement à celles issues de l'enquête Drees-Inserm de 2004 ; par ailleurs, nous ciblons les effets sur le marché du travail sur une échéance plus longue que celle des études.

La troisième réside dans le fait que nous considérons les individus en inactivité au moment du diagnostic, définis comme ceux qui ne cumulent aucun trimestre en emploi, maladie ou chômage. Cette mesure de l'inactivité est moins précise que l'observation directe de cet état au moment du diagnostic. De ce fait, nous ne sommes pas en mesure d'estimer précisément les sorties des situations de chômage ou d'emploi vers un état déclaré d'inactivité' ${ }^{16}$.

16. Notons que les travaux français sur l'enquête Drees-Inserm et qui étudient le retour à l'emploi à horizon de deux ans montrent que $10 \%$ des personnes en emploi au moment de leur diagnostic de cancer en 2002 étaient inactives (hors retraite) deux ans plus tard, $22 \%$ des personnes au chômage au moment de leur diagnostic de cancer en 2002 étaient inactives (hors retraite) et plus de $3 \%$ des personnes inactives au moment de leur diagnostic de cancer en 2002 étaient en emploi (voir Eichenbaum-Voline et al., 2008). 
Certains des résultats obtenus apparaissent cependant assez proches, notamment ceux qui signalent l'existence d'inégalités sociales de santé. Les études de Eichenbaum-Voline et al. (2008) et Joutard et al. (2012) établissent notamment que les probabilités de transition dépendent du statut socio-économique et de la sévérité du cancer. Dans ces études, pour les survivants du cancer avec les plus mauvais pronostics, la probabilité de rester en emploi est de $80 \%$ pour les hauts niveaux de statut socio-économique et de $63 \%$ pour les individus de plus faible statut. $\mathrm{Si}$ nous ne distinguons pas le pronostic du cancer, nous sommes en mesure de montrer que les individus appartenant aux quintiles de niveaux de salaires d'entrée sur le marché du travail les plus bas sont plus vulnérables sur le marché du travail face à la maladie que ceux appartenant aux quintiles de salaires supérieurs.

On doit cependant noter que les études menées sur l'enquête Drees-Inserm de 2004 concernent une population plus réduite que celle incluse dans notre travail qui s'appuie sur des données administratives.

De plus, dans l'enquête Drees-Inserm, un cinquième environ des personnes sélectionnées n'ont pu être interrogées en raison d'hospitalisations ou d'indisponibilités liées à la maladie. Ensuite, parmi les personnes finalement éligibles, un peu moins d'un quart n'a pas participé par refus de répondre ou impossibilité d'être contacté. Cette déperdition, liée pour une part aux soins, et pour une autre part au désir de ne pas participer, peut engendrer des différences dans l'appréciation des taux de retour à l'emploi en comparaison d'un échantillon administratif tel que le nôtre dont ne peuvent s'exclure les individus.

En outre dans l'estimation des transitions entre les différents statuts d'emploi pour les individus ayant eu un cancer, les auteurs s'exposent à un risque d'erreur. En effet, l'estimation d'un processus continu de Markov des transitions entre les différents statuts d'emploi pour les individus ayant eu un diagnostic de cancer (issus de l'enquête Drees-Inserm) et ceux n'ayant pas eu de diagnostic (le groupe de contrôle issu de l'enquête Emploi durant le même intervalle de temps) implique de corriger par un processus probabiliste la possibilité que les individus du groupe de contrôle peuvent avoir un cancer, ce qui n'est pas renseigné dans l'enquête Emploi, créant un risque d'erreur.

Au total, si nos résultats peuvent être confrontés à cette série d'études réalisées, ils ne peuvent être totalement comparés, du fait des spécificités inhérentes à chacune des études (données, méthodes), chacune ayant leurs avantages et limites.

Pour obtenir des comparaisons avec un horizon temporel plus long, nous devons nous tourner vers des travaux anglo-saxons. Cependant, très peu de ces travaux utilisent une base de données comparable à la nôtre. La plupart du temps, les travaux allient enquête sur échantillon de petite taille et données administratives (Bradley et al., 2002 sur le cancer du sein par exemple). Dans la revue de littérature menée par de Boer et Fings-Dresen (2009), sur tous les types de cancer, une baisse du taux d'emploi de plusieurs points est constatée après le diagnostic dans plusieurs études et variable selon le type de cancers. La diminution de l'employabilité est de 6 points pour des femmes atteintes d'un cancer du sein après cinq ans (Chirikos et al., 2002), entre 4 et 8 points dans un délai de deux à six ans après le diagnostic pour des patients âgés de 55 à 65 ans et atteints de cancer de tout type à l'exception des cancers de stade très avancé (Short et al., 2008).

Néanmoins, ces évaluations ne peuvent être strictement comparées du fait de la diversité des échantillons et des méthodes. Au regard des enseignements généraux, nos résultats apparaissent plus précis s'agissant des variables de résultat sur le marché du travail puisque nous disposons du nombre de jours passés en emploi, au chômage et en arrêts maladie. Le nombre de trimestres d'arrêts maladie consécutifs est élevé la première année suivant l'enregistrement administratif du cancer en ALD. L'accroissement du pourcentage d'individus avec au moins un trimestre en congé maladie est très important en raison des traitements médicaux reçus. S'agissant des effets du cancer à moyen terme, à partir de l'indicateur «nombre de trimestres validés dans la maladie », l'analyse montre une décroissance continue des trimestres validés pendant les quatre premières années après le cancer pour se stabiliser à un faible pourcentage de trimestres d'absences supplémentaires correspondant à un nombre moyen de quelques jours supplémentaires en arrêt de travail pour maladie. Enfin, nous identifions un effet positif mais d'importance moindre de la survenue du cancer sur la sortie du chômage après un an. Cet effet positif, probablement dû à l'obligation administrative de sortir de la situation de chômage indemnisé pour entrer dans celle d'arrêt maladie, devient ensuite nul pour les femmes et négatif pour les hommes (en raison d'un effet de substitution entre situations d'inactivité). 
Des effets propres au cancer peuvent expliquer ces résultats (notamment des effets liés aux séquelles des traitements). Il est probable également qu'ils se cumulent avec les effets plus traditionnels décrits par l'analyse économique : la maladie chronique éloigne régulièrement les individus du marché du travail qui doivent passer du temps à se soigner, ne serait-ce que du fait des contrôles réguliers ou des co-morbidités. Les préférences pour le temps non travaillé des travailleurs, supposées théoriquement exogènes, peuvent également se modifier.

Naturellement, plusieurs limites doivent être signalées. Outre celle liée à la définition retenue du cancer (cf. présentation de la base Hygie), les données ne nous permettent pas d'identifier le stade du cancer, la nature des traitements ou le degré de gravité (selon le grade impliquant la nature et la forme histologique des cellules des tumeurs, par exemple). À ce titre, la méconnaissance de l'état de santé du patient et l'utilisation exclusive de l'ALD cancer conduisent à mélanger potentiellement des personnes aux caractéristiques assez différentes, notamment eu égard à la localisation du cancer. D'autres travaux sont prévus et cela afin de travailler sur les cancers les plus prévalents dans notre base, notamment le cancer du sein (pour les femmes), de la prostate, des bronches et du poumon (pour les hommes), de la thyroïde (pour les femmes), du côlon.

La distinction selon l'âge médian permet de rendre compte, mais indirectement, de la localisation (le cancer du sein est un cancer qui peut survenir précocement, par exemple, et induit des traitements potentiellement agressifs). Cette distinction permet aussi d'isoler des dynamiques professionnelles plus favorables pour des personnes âgées de moins de 50 ans, notamment pour les hommes, sans doute à la fois plus orientés vers la carrière que les femmes d'âge similaire et soumis, à des âges jeunes, à des cancers potentiellement moins agressifs. Des phénomènes de discrimination de la part des employeurs peuvent enfin être en mesure d'expliquer le différentiel de résultat entre hommes et femmes.

\section{BIBLIOGRAPHIE}

Arrow K. J. (1973), « The Theory of Discrimination », dans O. Ashenfelter et A. Rees (eds.), Discrimination in Labor Markets, Princeton (NJ), Princeton University Press.

Becker G. S. (1957, 1971, 2 $2^{\mathrm{e}}$ éd.), The Economics of Discrimination, Chicago, University of Chicago Press.

Becker G. S.(1964, 1993, $3^{\mathrm{e}}$ éd.), Human Capital: A Theoretical and Empirical Analysis, with Special Reference to Education, Chicago, University of Chicago Press.

Belot A. et al. (2008), « Incidence et mortalité des cancers en France durant la période 1980-2005», Revue d'épidémiologie et de santé publique, $\mathrm{n}^{\circ} 56$, pp. 159-175.

Blinder V. S., Patil S., Thind A., Diamant A., Hudis C. A., Basch E. et Maly R. C. (2012), «Return to work in low-income Latina and non-Latina white breast cancer survivors: a 3-year longitudinal study », Cancer, vol. 118, $\mathrm{n}^{\circ} 6$, pp. 1664-1674.

De Boer A. G. et Frings-Dresen M. H.W. (2009), « Employment and the common cancers: return towork of cancer survivors ", Occupational Medicine, $\mathrm{n}^{\circ}$ 59, pp. 378-380.

De Boer A. G., Taskila T., Ojajarvi A., van Dijk F. J. et Verbeek J. H. (2009), « Cancer survivors and unemployment: A meta-analysis and meta-regression », JAMA, vol. 301, n ${ }^{\circ} 7$, pp. 753-762.

Bouvier G. et Jugnot S. (2013), « Les personnes ayant des problèmes de santé ou de handicap sont plus nombreuses que les autres à faire part de comportements stigmatisants », Économie et statistique, $\mathrm{n}^{\circ}$ 464-465-466, pp. 189-213.

Bradley C. J., Oberst K. et Schenk M. (2006), «Absenteeism from work: The experience of employed breast and prostate cancer patients in the months following diagnosis », Psychooncology, vol. $15, \mathrm{n}^{\circ} 8$, pp. 739-747.

Bradley C. J., Bednarek H. L. et Neumark D. (2002), «Breast cancer survival, work, and earnings », Journal of Health Economics, vol. 21, $\mathrm{n}^{\circ} 5$, pp. 757-779.

Chirikos T. N., Russell-Jacobs A. et Jacobsen P. B. (2002), «Functional impairment and the economic 
consequences of female breast cancer », Women and Health, vol. 36, $\mathrm{n}^{\circ}$ 1, pp. 1-20.

Chirikos T. N., Russell-Jacobs A., Cantor A. B. (2002), «Indirect economic effects of long-term breast cancer survival ». Cancer Practice, vol. 10, $\mathrm{n}^{\circ} 5$, pp. 248-255.

Coate S. et Loury G. (1993), « Will affirmative action policies eliminate negative stereotypes ?». American Economic Review, vol. 83, $\mathrm{n}^{\circ}$ 5, pp. $1220-1240$.

Cooper A. F., Hankins M., Rixon L., Eaton E. et Grunfeld E. (2012), « Distinct Work-related, clinical and Psychological factors predict return to work following treatment in four different cancer types "), Psycho-oncology, doi : 10.1002/pon.3049.

Crompton R. (1999), Restructuring gender relations and employment: The decline of the male breadwinner, Oxford University Press.

Damkjaer L. H., Deltour, Suppli I., Palm N., Kroman J., Johansen N. T., Dalton C. et Oksbjerg S. (2011), « Breast cancer and early retirement: Associations with disease characteristics, treatment, comorbidity, social position and participation in a six-day rehabilitation course in a register-based study in Denmark », Acta Oncologica, vol. 50, $\mathrm{n}^{\circ} 2$, pp. 274-81.

Danzon A., Le Moal J., Chérié-Challine L. et Viso A. C. (2012), « La surveillance épidémiologique des cancers en France : outils actuels et perspectives », Bulletin épidémiologique hebdomadaire, 31 janvier, $\mathrm{n}^{\circ}$ 5-6, pp. 54-58.

Datta Gupta N. et Larsen M. (2010), « The impact of health on individual retirement plans: Self-reported versus diagnostic measures », Health Economics, vol. 19, n 7, pp. 792-813.

Drolet M., Maunsell E., Mondor M., Brisson C., Brisson J., Mâsse B. et Deschênes L. (2005), «Work absence after breast cancer diagnosis: A population-based study », Canadian Medical Association Journal, vol. 173, n 7, pp. 765-771.

Duguet E. et Le Clainche C. (2012a), « Une évaluation de l'impact de l'aménagement des conditions de travail sur la reprise du travail après un cancer », Document de travail CEE, $\mathrm{n}^{\circ} 159$, à paraître dans La Revue économique.

Duguet E. et Le Clainche C. (2012b), «The Impact of Health Events on Individual Labor Market Histories: The Message from Difference-
in-Differences with Exact Matching », LAMETA DR $n^{\circ}$ 2012-08.

Eichenbaum-Voline S., Malavolti L., Paraponaris A. et Ventelou B. (2008), «Cancer et activité professionnelle », La Revue de l'OFCE, $\mathrm{n}^{\circ}$ 104, pp. 105-134.

Fantoni-Quinton S., Peugniez C., Duhamel A., Skrzypczak J., Frimat P. et Leroyer A. (2010), « Factors related to return to work by women with breast cancer in Northern France ", Journal of Occupational Rehabilitation, $\mathrm{n}^{\circ} 20$, pp. 49-58.

Feuerstein M., Todd B. L., Moskowitz M. C., Bruns G. L., Stoler M. R., Nassif T. et Yu X. (2010), «Work in cancer survivors: A model for practice and research », Journal of Cancer survivorship, research and practice, $\mathrm{n}^{\circ} 4$, pp. 415-437.

Fougère D. (2010), «Les méthodes économétriques d'évaluation», Revue française des Affaires sociales, $\mathrm{n}^{\circ} 1-2$, pp. 105-128.

Govillot S. et Rey M. (2013), «Rechercher et retrouver un emploi après 55 ans », dans Emploi et salaires - Insee Références, pp. 27-40.

Gregg P. et Tominey E. (2005), « The Wage Scar from Youth Unemployment », Labour Economics, $\mathrm{n}^{\circ} 12$, pp. 487-509.

Grosclaude P., Dentan C., Trétarre B., Velten M., Fournier E. et Molinié F. (2012), «Utilité des bases de données médico-administratives pour le suivi épidémiologique des cancers. Comparaison avec les données des registres au niveau individuel », Bulletin épidémiologique hebdomadaire, 31 janvier, $n^{\circ}$ 5-6, pp. 63-67.

Grossman M. (1972), « On the Concept of Health Capital and the Demand for Health », Journal of Political Economy, vol. 80, n 2, pp. 223-255.

Grossman M. (2000), "The human capital model », dans A. J. Culyer et J. P. Newhouse (eds.), Handbook of Health Economics, édition 1, vol. 1, chap. 7, Elsevier, pp. 347-408.

Heckman J.-J. et Borjas G. (1980), «Does Unemployment Cause Future Unemployment? Definitions, Questions and Answers from a Continuous Time Model of Heterogeneity and State Dependence », Economica, n 47, pp. 247-283.

INCa (2015), Les cancers en France. Edition 2014. Collection État des lieux et des connaissances, INCa, Boulogne-Billancourt. 
Johnsson A., Fornander T., Rutqvist L. E. et Olsson M. (2011), "Work Status and Life Changes in the First Year after Breast Cancer Diagnosis », Work, n 38 , pp. 337-346.

Joutard X., Paraponaris A., Sagaon-Teyssier L. et Ventelou B. (2012), «A Continuous-time Markov Model for Transitions Between Employment and Non-employment: The Impact of a Cancer Diagnosis », Annals of Economics and Statistics, vol. 107-108, pp. 239-266.

Lindbohm M.-L., Kuosma E., Taskila T., Hietanen P., Carlsen K., Gudbergsson S. et Gunnarsdottir H., (2011), « Cancer as the cause of changes in work situation ", Psycho-oncology, vol. 20, pp. 805-812.

Malavolti L., Paraponaris A. et Ventelou B. (2008), « La reprise du travail après un diagnostic de cancer : un processus distinct entre hommes et femmes "), dans Le Corroller-Soriano, Malavolti et Mermilliod (éds.), La vie deux ans après le diagnostic de cancer, Drees-Inserm, Coll. Études et Statistiques, La Documentation française, pp. 259-270.

Marino P., Sagaon-Teyssier L., Malavolti L. et Le Corroller-Soriano A. G. (2013), « Sex Differences in the Return To Work Process of Cancer Survivors 2 Years After Diagnosis: Results From A Large French Population-Based Sample », Journal of Clinical Oncology, vol. 31, pp. 1-10.

Mehnert A. (2011), «Employment and workrelated issues in cancer survivors », Critical Reviews in Oncology Hematology, ${ }^{\circ}$ 77, pp. 109-130.

Moran J. R., Short P. F. et Hollenbeak C. S. (2011), «Long-term Employment effects of Surviving Cancer», Journal of Health Economics, vol. 30, n 3 , pp. 505-514.

Mujahid M. S., Janz N. K., Hawley S. T., Griggs J. J., Hamilton A. S., Graff J. et Katz S. J. (2011), «Racial/ethnic differences in job loss for women with breast cancer», Journal of
Cancer survivorship, Research and Practice, $\mathrm{n}^{\circ} 5$, pp. 102-111.

Oberst K., Bradley C. J., Gardiner J. C., Schenk M. et Given C. W. (2010), « Work task disability in employed breast and prostate cancer patients ». Journal of Cancer survivorship, Research and Practice, vol. 4, n 4, pp. 322-330.

Paraponaris A., Teyssier L. S. et Ventelou B. (2010), « Job Tenure and Self-reported Workplace Discrimination For Cancer Survivors 2 Years After Diagnosis: Does employment legislation matter? », Health Policy, vol. 98, n 2-3, pp. 144-155.

Park J. H., Park E. C., Park J. H., Kim S. G. et Lee S. Y. (2008), « Job loss and re-employment of cancer patients in Korean employees: Anationwide retrospective cohort study », Journal of Clinical Oncology, vol. 26, n 8, pp. 1302-1309.

Phelps E. (1972), «The statistical theory of racism and sexism », American Economic Review, $\mathrm{n}^{\circ} 62$, pp. 659-661.

Quinlan E., Thomas-MacLean R., Hack T., Kwan W., Miedema B., Tatemichi S., Towers A. et Tilley A. (2009), « The impact of breast cancer among Canadian women: Disability and productivity »,Work, vol. 34, n 3, pp. 285-296.

Short P. F., Vasey J. J. et Belue R. (2008), « Work disability associated with cancer survivorship and other chronic conditions ", Psycho-Oncology, vol. 17, $\mathrm{n}^{\circ} 1$, pp. 91-97.

Taskila-Abrandt T., Pukkala E., Martikainen R., Karjalainen A. et Hietanen P. (2005), «Employment status of Finnish cancer patients in $1997 »$, Psycho-Oncology, vol. 14, $\mathrm{n}^{\circ} 3$, pp. 221-226.

Tomasetti C. et Vogelstein B. (2015), « Variation in cancer risk among tissues can be explained by the number of stem cell divisions », Science 2 January 2015, vol. 347, n 6217, pp. 78-81. 